\title{
Ax-Kochen-Ershov Principles for Valued and Ordered Vector Spaces*
}

\author{
Franz-Viktor and Salma Kuhlmann
}

17. 1.1996

\begin{abstract}
We study extensions of valued vector spaces with variable base field, introducing the notion of disjointness and valuation disjointness in this setting. We apply the results to determine the model theoretic properties of valued vector spaces (with variable base field) relative to that of their skeletons. We study the model theory of the skeletons in special cases. We apply the results to ordered vector spaces with compatible valuation.
\end{abstract}

\section{Introduction}

Let $K$ be a field, $V$ a $K$-vector space and $\Gamma$ a totally ordered set with $\infty$ as its last element. A surjective map

$$
v: V \longrightarrow \Gamma
$$

is a valuation on $V$ (and $\left({ }_{K} V, v\right)$ is a valued vector space) if for all $x, y \in V$ and $k \in K$, the following holds:

(i) $v(x)=\infty$ if and only if $x=0$,

(ii) $v(x-y) \geq \min \{v(x), v(y)\} \quad$ (ultrametric triangle law).

In this paper, we will only consider valued vector spaces with value preserving scalar multiplication, which means that they satisfy:

(iii) $v(k x)=v(x)$ if $k \neq 0$.

This condition is crucial for the results of this paper.

We call $v(V):=\Gamma$ the value set of $\left({ }_{K} V, v\right)$. Let $\gamma \in \Gamma$ and put

$$
\begin{aligned}
V^{\gamma} & =\{x \in V ; v(x) \geq \gamma\} \\
V_{\gamma} & =\{x \in V ; v(x)>\gamma\} .
\end{aligned}
$$

Then $V^{\gamma}, V_{\gamma}$ are $K$-subspaces (for $\gamma \neq \infty$ ) satisfying $V_{\gamma} \subset V^{\gamma} \subset V$. We put

$$
B(V, \gamma)=V^{\gamma} / V_{\gamma}, B(V, \infty)=0
$$

\footnotetext{
${ }^{*}$ This paper was written while the second author was supported by the Deutsche Forschungsgemeinschaft.

This paper represents some results from the second author's doctoral thesis.
} 
The $K$-vector space $B(V, \gamma)$ is called the component corresponding to $\gamma$. The skeleton of $\left({ }_{K} V, v\right)$ is the system $S\left({ }_{K} V, v\right):=(K, v(V),\{B(V, \gamma) ; \gamma \in v(V)\})$. We introduce a coefficient map $\pi^{V}$ as follows. For every $\gamma \in \Gamma$, the coefficient map corresponding to $\gamma$ is the canonical homomorphism

$$
\pi^{V}(\gamma,-): V^{\gamma} \longrightarrow B(V, \gamma) \text { defined by } \pi^{V}(\gamma, x)=x+V_{\gamma} .
$$

The aim of our paper is to study extensions of valued vector spaces and their model theory. For this, we do not want to fix the base field. Throughout this paper, we let $K$ be an arbitrary field. If $F$ is a subfield of $K$ and $V$ is a $K$-vector space, then in a canonical manner, $V$ is also an $F$-vector space. We write ${ }_{K} V$ and ${ }_{F} V$ to distinguish these two structures. We write ${ }_{F} U \subset{ }_{K} V$ if $F$ is a subfield of $K$ and ${ }_{F} U$ is an $F$-subspace of ${ }_{F} V$. If $X$ is a subset of $V$, then we write ${ }_{F}\langle X\rangle$ for the $F$-subspace of $V$ generated by $X$; hence, ${ }_{F}\langle X\rangle \subset{ }_{K} V$. We will frequently write $\langle X\rangle$ for ${ }_{K}\langle X\rangle$.

In Section 3, we study extensions of the form ${ }_{F} U \subset{ }_{K} V$, also in the presence of a valuation. The only chance to have a control on such extensions is given if ${ }_{F} U$ is disjoint (respectively, valuation disjoint) from $K$ in ${ }_{K} V$, that is, if $F$-independent (respectively, $F$-valuation independent) elements in $U$ remain $K$ independent (respectively, $K$-valuation independent) in $V$. We give criteria and show transitivity for these properties. We prove that valuation disjointness of a valued vector space is equivalent to disjointness for all of its components (Proposition 3.12). Further, we describe $\langle U\rangle$ in this case: as a valued $K$-vector space, it is uniquely determined by the valued $F$-vector space ${ }_{F} U$ (Proposition 4.3). Moreover, its components are just the subspaces generated by the corresponding components of $U$ (Proposition 3.10), and its value set is equal to that of $U$. Finally, we combine the conditions of valuation disjointness and that of niceness to get the notion of correct extensions. We investigate the properties of these extensions, and show results analogous to those one gets for nice subspaces.

In Section 5, we apply the algebraic results to study the model theory of valued vector spaces. The motivating question is: to which extend do the model theoretic properties of the skeleton determine those of the valued vector space? In [K3], the theory of valued vector spaces and the theory of skeletons are formalised in first order languages. In principle, we have the following two possibilities for the formalisation of vector spaces:

a) The base field $K$ is fixed and we work in a one-sorted language $\mathcal{L}^{K}$ which contains function symbols for scalar multiplication (one for each element in $K$ ).

b) The base field is not fixed and we work in a two-sorted language $\mathcal{L}$ which contains one binary function symbol for the scalar multiplication of an element of the field sort with an element of the space sort.

In both cases, the valuation can e.g. be formalised by a binary predicate expressing that $v(x) \geq v(y)$. Adjoining this predicate, we obtain languages $\mathcal{L}_{\mathrm{V}}^{K}$ and $\mathcal{L}_{\mathrm{V}}$. Corresponding to these two versions, the skeletons can be formalised in a language $\mathcal{L}_{\mathrm{S}}^{K}$ with fixed base field $K$, or in a language $\mathcal{L}_{\mathrm{S}}$ with variable base field. We will not carry out the formalisation or give the axiom systems here; for a detailed description, we refer the reader to [K3]. Let us say in advance that all of our model theoretic results remain valid if we formalise the valued vector spaces in a 
many-sorted language $\mathcal{L}_{\mathrm{VS}}=\mathcal{L}_{\mathrm{V}} \cup \mathcal{L}_{\mathrm{S}} \cup\{\pi\}$ so as to include their skeletons. Then we can talk about the valuation $v$ as a function from the space sort onto the value set sort, and of the coefficient map $\pi$ as a function from the space sort onto the components sort.

If $\mathcal{M}$ and $\mathcal{N}$ are structures for an arbitrary language $\mathcal{L}$, then $\mathcal{M} \prec \exists \mathcal{N}$ stands for " $\mathcal{M}$ is existentially closed in $\mathcal{N}$ ". This means that every first order existential sentence with parameters from $\mathcal{M}$ which holds in $\mathcal{N}$, also holds in $\mathcal{M}$.

We show the following Ax-Kochen-Ershov Principles for valued vector spaces:

Theorem 1.1 1) Suppose that $\left({ }_{F} U, v\right) \subset\left({ }_{K} V, v\right)$ is an extension of valued vector spaces. Then $\left({ }_{F} U, v\right) \prec_{\exists}\left({ }_{K} V, v\right)$ in $\mathcal{L}_{\mathrm{V}}$ if and only if $S\left({ }_{F} U, v\right) \prec_{\exists} S\left({ }_{K} V, v\right)$ in $\mathcal{L}_{\mathrm{S}}$.

2) The same holds with $\prec$ ("elementary substructure") in the place of " $\exists_{\exists}$ ".

3) Let $\left({ }_{K} V, v\right)$ and $\left({ }_{K^{\prime}} V^{\prime}, v^{\prime}\right)$ be valued vector spaces. Then $\left({ }_{K} V, v\right) \equiv\left({ }_{K^{\prime}} V^{\prime}, v^{\prime}\right)$ ("elementarily equivalent") in $\mathcal{L}_{\mathrm{V}}$ if and only if $S\left({ }_{K} V, v\right) \equiv S\left({ }_{K}, V^{\prime}, v^{\prime}\right)$ in $\mathcal{L}_{\mathrm{S}}$.

4) Let $(V, v)$ and $\left(V^{\prime}, v^{\prime}\right)$ be valued $K$-vector spaces with common valued $K$-subspace $(U, v)$. Then $(V, v) \equiv\left(V^{\prime}, v^{\prime}\right)$ over $(U, v)$ in $\mathcal{L}_{\mathrm{V}}^{K}$ if and only if $S(V, v) \equiv S\left(V^{\prime}, v^{\prime}\right)$ over $S(U, v)$ in $\mathcal{L}_{\mathrm{S}}^{K}$.

For every field $K$, every totally ordered set $\Gamma$ and every collection $\left\{B_{\gamma} ; \gamma \in \Gamma\right\}$ of $K$-vector spaces there is a valued $K$-vector space with skeleton $\left(K, \Gamma,\left\{B_{\gamma} ; \gamma \in \Gamma\right\}\right)$ (we can take the Hahn sum or the Hahn product of the spaces $B_{\gamma}, \gamma \in \Gamma$, cf. [K1]). From this and Theorem 1.1 it follows that if $\mathcal{V}$ is an $\mathcal{L}_{\mathrm{V}}$-elementary class of valued vector spaces (or an $\mathcal{L}_{\mathrm{V}}^{K}$-elementary class of valued $K$-vector spaces), then the class of all of their skeletons is $\mathcal{L}_{\mathrm{S}}$-elementary (or $\mathcal{L}_{\mathrm{S}}^{K}$-elementary).

Corollary 1.2 An $\mathcal{L}_{\mathrm{V}}$-elementary class of valued vector spaces is complete (or model complete) if and only if the $\mathcal{L}_{\mathrm{S}}$-elementary class of their skeletons has the same property. An $\mathcal{L}_{\mathrm{V}}^{K}$-elementary class of valued $K$-vector spaces is complete (or model complete, or admits quantifier elimination) if and only if the $\mathcal{L}_{\mathrm{S}}^{K}$-elementary class of their skeletons has the same property.

Analysing some conditions for a class of skeletons to be complete or model complete (Section 5.3), we will prove:

Theorem 1.3 An elementary class of valued vector spaces with dense value set without endpoints and infinite-dimensional components is complete if and only if the elementary class of their base fields is complete.

The class of all valued $K$-vector spaces with dense value set without endpoints and infinite components is complete and admits quantifier elimination.

If we enrich our languages of valued vector spaces and of skeletons by additional predicates, then we obtain analogues of Theorem 1.1 and its consequences, provided that the predicates on the skeleton are definable from the predicates on the spaces and vice versa (cf. Section 5.4). We wish to apply this to the theory of ordered vector spaces (with compatible valuation).

If $\left({ }_{K} V,<\right)$ is an ordered $K$-vector space (cf. [K1]), then a valuation on $V$ is said to be compatible with the order if for all $x, y \in V, 0 \leq x \leq y$ implies that $v x \geq v y$ (in other words, $v: V \leq 0=\{x \in V ; x \leq 0\} \rightarrow v(V)$ is order preserving). This property is obviously elementary in both languages $\mathcal{L}_{\mathrm{V}}^{K}$ and $\mathcal{L}_{\mathrm{V}}$. We define

$$
x \sim_{K} y: \Leftrightarrow \exists k \in K: k|x| \geq|y| \wedge k|y| \geq|x|,
$$


where $|x|=\max \{x,-x\}$. If $x \sim_{K} y$, then we say that $x$ and $y$ are $K$-archimedean equivalent (if $K$ is an archimedean field, then this just means that $x$ and $y$ are archimedean equivalent); cf. [K1]. Our general hypothesis that the scalar multiplication is value preserving means that $x \sim_{K} y \Rightarrow v(x)=v(y)$. If also the converse holds, that is, if $v$ induces an order isomorphism of the ordered set of all $K$-archimedean classes of $V$ onto the value set, then $v$ is called the natural valuation of $(V,<)$. Note that the fact that $v$ is the natural valuation cannot be expressed by an elementary sentence in the one-sorted language of valued $K$-vector spaces with an order. But in the two-sorted language of valued vector spaces with an order, " $x \sim_{K} y$ " and hence also the following is an elementary formula:

$$
x \sim_{K} y \leftrightarrow v(x)=v(y) .
$$

Every compatible valuation on an ordered $K$-vector space $\left({ }_{K} V,<\right)$ induces the structure of an ordered $K$-vector space on every component via:

$$
0<x \Rightarrow 0<\pi^{V}(v(x), x) .
$$

We will then speak of the ordered skeleton $S\left({ }_{K} V,<, v\right)$. On the other hand, an ordered $K$-vector space structure on every component $B_{\gamma}$ of a valued vector space lifts to a unique ordered $K$-vector space structure on $V$ via the converse of (1). So if we set $\mathcal{L}_{\mathrm{OV}}:=\mathcal{L}_{\mathrm{V}} \cup\left\{<_{V}\right\}$ and $\mathcal{L}_{\mathrm{OS}}:=\mathcal{L}_{\mathrm{S}} \cup\left\{<_{B}\right\}$, and if we add the axiom

$$
0<_{V} x \leftrightarrow 0<_{B} \pi(v(x), x)
$$

to the theory of ordered vector spaces with compatible valuation and coefficient map, formalised in the language $\mathcal{L}_{\text {OVS }}:=\mathcal{L}_{\text {OV }} \cup \mathcal{L}_{\text {OS }} \cup\{\pi\}$, then $<_{V}$ and $<_{B}$ become interdefinable. So we obtain from Theorem 5.11:

Theorem 1.4 Suppose that $\left({ }_{F} U,<, v\right) \subset\left({ }_{K} V,<, v\right)$ is an extension of ordered vector spaces with compatible valuation. Then $\left({ }_{F} U,<, v\right) \prec_{\exists}\left({ }_{K} V,<, v\right)$ in $\mathcal{L}_{\mathrm{OV}}$ if and only if $S\left({ }_{F} U,<, v\right) \prec_{\exists} S\left({ }_{K} V,<, v\right)$ in $\mathcal{L}_{\mathrm{OS}}$.

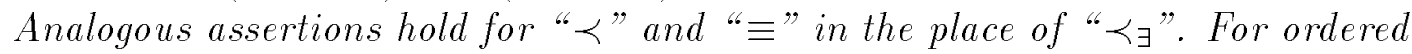
$K$-vector spaces with compatible valuation (with analogously defined languages $\mathcal{L}_{\mathrm{OV}}^{K}$ and $\mathcal{L}_{\mathrm{OS}}^{K}$ in the place of $\mathcal{L}_{\mathrm{OV}}$ and $\mathcal{L}_{\mathrm{OS}}$ ), we also have the analogue to assertion 4 ) of Theorem 1.1 .

Corollary 1.5 An $\mathcal{L}_{\mathrm{OV}}$-elementary class of ordered vector spaces with compatible valuation is complete (or model complete) if and only if the $\mathcal{L}_{\mathrm{OS}}$-elementary class of their ordered skeletons has the same property. An $\mathcal{L}_{\mathrm{OV}}^{K}$-elementary class of ordered $K$-vector spaces with compatible valuation is complete (or model complete, or admits quantifier elimination) if and only if the $\mathcal{L}_{\mathrm{OS}}^{K}$-elementary class of their ordered skeletons has the same property.

Now let $(K,<)$ be an ordered field. An ordered $(K,<)$-vector space then is an ordered $K$-vector space which in addition satisfies the axiom scheme

$$
0<x \rightarrow 0<k x \quad(k \in K, k>0)
$$

where " $<$ " stands for " $<_{V}$ " if we deal with the ordered vector space $V$, and for " $<_{B}$ " if we deal with its ordered components. For the convenience of the reader, we prove the following theorem of L. v. d. Dries (cf. [D]) in Section 5.5. 
Theorem 1.6 Let $(K,<)$ be an ordered field. The elementary class of ordered $(K,<)$-vector spaces is complete and admits quantifier elimination.

From a corresponding result for skeletons and Corollary 1.5, we will deduce:

Theorem 1.7 An elementary class of ordered $(K,<)$-vector spaces with compatible valuation is complete (or model complete, or admits quantifier elimination) if and only if the elementary class of their value sets has the same property.

In a subsequent paper, we will give applications of Corollary 1.5 to the case of ordered vector spaces with compatible valuation and variable ordered base field.

For the proof of the above results, we do not need our results on correct extensions. But they are needed in [K3] to show that the infinitary properties of the skeleton determine the infinitary properties of a valued vector space.

For the case of scalar multiplication which does not preserve the value (as it is the case in a valued field extension $(L, v)$ of $(K, v)$, viewed as valued $(K, v)$-vector space), see the results of [D].

\section{Preliminaries on valued vector spaces}

We first recall briefly some definitions and results from [K1; Section 2] and [K2], but we refer the reader to these papers for details and proofs. In the following, let

$$
(U, v) \subset(V, v)
$$

be an extension of valued $K$-vector spaces, that is, the restriction of the valuation of $V$ coincides with the valuation of $U$. Then we have a corresponding extension of skeletons, that is, $v(U) \subset v(V)$ and $B(U, \gamma)$ is (canonically isomorphic to) a subspace of $B(V, \gamma)$ for every $\gamma \in v(V)$ (we set $B(U, \gamma)=0$ if $\gamma \notin v(U)$ ). If this extension of skeletons is trivial, then the extension (3) is called immediate. Equivalently, (3) is immediate if and only if for every $x \neq 0$ in $V$ there is some $z \in U$ such that $v(x+z)>v(x)$. On the other hand, a set $\mathcal{B} \subset V$ is called $(K-)$ valuation independent over $U$ if $0 \notin \mathcal{B}$ and

$$
v\left(\sum_{x \in \mathcal{B}} k_{x} x+z\right)=\min _{\left\{x \in \mathcal{B} ; k_{x} \neq 0\right\}}\{v(x), v(z)\}
$$

holds for all $z \in U$ and $k_{x} \in K$ such that $k_{x}=0$ except for a finite number of $x$ 's. (Note that in particular, the sum can only be zero if $z$ and all $k_{x}$ are zero; that is, $\mathcal{B} \subset V$ is $(K-)$ independent over $U$.) If in addition $\mathcal{B}$ is a $K$-basis of $V$ over $U$, then $\mathcal{B}$ is called a $(K-)$ valuation basis of $V$ over $U$.

For every subset $\mathcal{B} \subset V$ and $\gamma \in v(V)$, we define $\mathcal{B} \gamma:=\{b \in \mathcal{B} ; v(b)=\gamma\}$; so the non-empty among the sets $\mathcal{B} \gamma, \gamma \in v(V)$ form a partition of $\mathcal{B}$. We need the following fact (cf. [K1], Proposition 2.10 and Corollary 2.11):

Proposition 2.1 $A$ subset $\mathcal{B} \subset V$ is valuation independent over $U$ if and only if for every $\gamma \in v(V)$, the subset

$$
\pi^{V}(\gamma, \mathcal{B}):=\left\{\pi^{V}(\gamma, x) ; x \in \mathcal{B} \gamma\right\}
$$

of $B(V, \gamma)$ is $K$-independent over $B(U, \gamma)$. Further, $\mathcal{B}$ is maximal with this property if and only if $\pi^{V}(\gamma, \mathcal{B})$ is a basis of $B(V, \gamma)$ over $B(U, \gamma)$, for every $\gamma \in v(V)$. 
We say that the subspace $U$ of $V$ is nice in $V$ if for all $x \in V$, the subset $v(x+U)=\{v(x+z) ; z \in U\}$ of $v(V)$ admits a maximum. This is equivalent to: for all $x \in V \backslash U$, the subspace $U+K x$ admits a valuation basis over $U$ (cf. [K2], Lemma 2.6). Note that the trivial subspace 0 is nice in $V$. For the following results, see [K2] (Corollary 3.4, Corollary 3.7, Theorem 3.9 and Corollary 2.9):

Theorem 2.2 Let $U$ be nice in $V$.

a) If $\operatorname{dim} V / U \leq \aleph_{0}$, then $V$ admits a valuation basis over $U$.

b) If $U \subset V^{\prime} \subset V$ and $V^{\prime} U$ is finite-dimensional, then $V^{\prime}$ is again nice in $V$.

In particular, if $\operatorname{dim} V \leq \aleph_{0}$, then $V$ admits a valuation basis over every subspace of finite dimension.

Theorem 2.3 Let $\mathcal{B} \subset V$ be maximal valuation independent over $U$. If $V^{\prime}$ is the vector space generated by $\mathcal{B}$ over $U$, then $U$ is nice in $V^{\prime}$, and $V^{\prime} \subset V$ is an immediate extension.

\section{Extending the base field}

\subsection{Linear disjointness}

Throughout this section, we assume that

$$
{ }_{F} W \subset{ }_{F} U \subset{ }_{K} V \text {. }
$$

We say that $U$ is (linearly) disjoint from $K$ in $V$ over $W$ if every subset $X \subset U$ which is $F$-independent over $W$, remains $K$-independent over $\langle W\rangle$ in ${ }_{K} V$. (Note that it is sufficient to check this condition for finite $X$.) Further, $U$ is (linearly) disjoint from $K$ in $V$ if $U$ is disjoint from $K$ in $V$ over 0 .

Remark 3.1 1) Note that $U$ is disjoint from $K$ in $V$ over $W$ if and only if $U$ is disjoint from $K$ in $\langle U\rangle$ over $W$.

2) Let ${ }_{F} U \subset{ }_{K} V^{\prime}$ and $f:{ }_{K} V \simeq{ }_{K} V^{\prime}$ be an isomorphism over $U$. Then $U$ is disjoint from $K$ in $V$ over $W$ if and only if $U$ is disjoint from $K$ in $V^{\prime}$ over $W$.

3) Suppose that $X \subset U$ is $K$-independent. If $k_{x} \in K$, equal to zero for almost all $x \in X$, but not all in $F$, then

$$
\sum_{x \in X} k_{x} x \in V \backslash U
$$

Proposition 3.2 Suppose that $\langle W\rangle \subset{ }_{K} W^{\prime} \subset{ }_{K} V$. The following are equivalent:

1) every $X \subset U$ which is $F$-independent over $W$, remains $K$-independent over $W^{\prime}$.

2) every $F$-basis of $U$ over $W$ remains a $K$-basis of $\langle U\rangle+W^{\prime}$ over $W^{\prime}$.

3) there is an $F$-basis of $U$ over $W$ which remains $K$-independent in $V$ over $W^{\prime}$.

Proof: 1$) \Rightarrow 2$ ): Every $F$-basis of $U$ over $W$ remains a system of $K$-generators of $\langle U\rangle$ over $\langle W\rangle$ and hence also of $\langle U\rangle+W^{\prime}$ over $W^{\prime}$. By 1$)$, it remains $K$-independent over $W^{\prime}$.

$2) \Rightarrow 3$ ): Trivial. 
$3) \Rightarrow 1$ ): Let $\mathcal{B}$ be an $F$-basis of $U$ over $W$ which remains $K$-independent in $V$ over $W^{\prime}$. Assume $x_{1}, \ldots, x_{r} \in U$ to be $F$-independent over $W$. Write

$$
x_{i}=\sum_{j=1}^{n_{i}} k_{i j} b_{i j}, \quad 1 \leq i \leq r
$$

with $k_{i j} \in F$ and $b_{i j} \in \mathcal{B}$. Set

$$
W_{0}:={ }_{F}\left\langle b_{i j} ; 1 \leq i \leq r, 1 \leq j \leq n_{i}\right\rangle+W .
$$

We complete $\left\{x_{1}, \ldots, x_{r}\right\}$ to an $F$-basis $\left\{x_{1}, \ldots, x_{m}\right\}$ of $W_{0}$ over $W$. By hypothesis, the $F$-basis $\left\{b_{i j} ; 1 \leq i \leq r, 1 \leq j \leq n_{i}\right\}$ of $W_{0}$ over $W$ remains $K$-independent in $V$ over $W^{\prime}$. So $\operatorname{dim}_{K}\left(\left\langle W_{0}\right\rangle+W^{\prime}\right) / W^{\prime}=m$. Consequently, $\left\{x_{1}, \ldots, x_{m}\right\}$ and also $\left\{x_{1}, \ldots, x_{r}\right\}$ remains $K$-independent in $V$ over $W^{\prime}$.

With $W^{\prime}=\langle W\rangle$, we obtain:

Corollary 3.3 The following are equivalent:

1) $U$ is disjoint from $K$ in $V$ over $W$

2) every $F$-basis of $U$ over $W$ remains a $K$-basis of $\langle U\rangle$ over $\langle W\rangle$.

3) there is an $F$-basis of $U$ over $W$ which remains $K$-independent in $V$ over $\langle W\rangle$.

In particular, if $\operatorname{dim}_{F} U / W$ is finite, then $U$ is disjoint from $K$ in $V$ over $W$ if and only if $\operatorname{dim}_{F} U / W=\operatorname{dim}_{K}\langle U\rangle /\langle W\rangle$.

\section{Proposition 3.4 (Transitivity)}

Suppose that ${ }_{F} U \subset{ }_{K^{\prime}} V^{\prime} \subset{ }_{K} V$. If $U$ is disjoint from $K^{\prime}$ in $V^{\prime}$ over $W$ and $V^{\prime}$ is disjoint from $K$ in $V$ over ${ }_{K^{\prime}}\langle W\rangle$, then $U$ is disjoint from $K$ in $V$ over $W$. Conversely, if $U$ is disjoint from $K$ in $V$ over $W$, then $U$ is disjoint from $K^{\prime}$ in $V^{\prime}$ over $W$; if in addition $V^{\prime}={ }_{K^{\prime}}\langle U\rangle$, then $V^{\prime}$ is disjoint from $K$ in $V$ over ${ }_{K^{\prime}}\langle W\rangle$.

Proof: The first assertion follows from the fact that $\langle W\rangle={ }_{K}\left\langle{ }_{K^{\prime}}\langle W\rangle\right\rangle$.

For the converse, let $\mathcal{B}$ be an $F$-basis of $U$ over $W$. By hypothesis, $U$ is disjoint from $K$ in $V$ over $W$, so by Corollary $3.3, \mathcal{B}$ remains a $K$-basis of $\langle U\rangle$ over $\langle W\rangle$. But $\mathcal{B}$ is also a system of $K^{\prime}$-generators of ${ }_{K^{\prime}}\langle U\rangle$ over ${ }_{K^{\prime}}\langle W\rangle$. Hence $\mathcal{B}$ is a $K^{\prime}$-basis of ${ }_{K^{\prime}}\langle U\rangle$ over ${ }_{K^{\prime}}\langle W\rangle$ which remains $K$-independent in ${ }_{K} V$ over $\langle W\rangle={ }_{K}\left\langle{ }_{K^{\prime}}\langle W\rangle\right\rangle$. Again by Corollary 3.3, it follows that $U$ is disjoint from $K^{\prime}$ in $V^{\prime}$ over $W$ and that ${ }_{K^{\prime}}\langle U\rangle$ is disjoint from $K$ in $V$ over ${ }_{K^{\prime}}\langle W\rangle$.

The following proposition states a different form of transitivity:

Proposition 3.5 Suppose now that ${ }_{F} U \subset{ }_{F} V^{\prime} \subset{ }_{K} V$. If $V^{\prime}$ is disjoint from $K$ in $V$ over $U$ and $U$ is disjoint from $K$ in $V$ over $W$, then $V^{\prime}$ is disjoint from $K$ in $V$ over $W$.

Proof: By Corollary 3.3, it suffices to show that there exists an $F$-basis of $V^{\prime}$ over $W$ which remains $K$-independent in $V$ over $\langle W\rangle$. Again by Corollary 3.3 and by hypothesis, let $\mathcal{B}$ be an $F$-basis of $U$ over $W$ which remains $K$-independent in $V$ over $\langle W\rangle$, and $\mathcal{B}^{\prime}$ an $F$-basis of $V^{\prime}$ over $U$ which remains $K$-independent in $V$ 
over $\langle U\rangle$. Then $\mathcal{B} \cup \mathcal{B}^{\prime}$ is the required basis: indeed, it is an $F$-basis of $V^{\prime}$ over $W$ since

$$
V^{\prime}={ }_{F}\left\langle\mathcal{B}^{\prime}\right\rangle \oplus U={ }_{F}\left\langle\mathcal{B}^{\prime}\right\rangle \oplus{ }_{F}\langle\mathcal{B}\rangle \oplus W={ }_{F}\left\langle\mathcal{B} \cup \mathcal{B}^{\prime}\right\rangle \oplus W
$$

Moreover, $\mathcal{B} \cup \mathcal{B}^{\prime}$ is $K$-independent in $V$ over $\langle W\rangle$ since $\mathcal{B}^{\prime}$ is $K$-independent in $V$ over $\langle U\rangle$ and $\mathcal{B}$ is $K$-independent in $V$ over $\langle W\rangle$ (cf. [K2; Lemma 2.1]).

\subsection{Valuation Disjointness}

Now we assume in addition to $(4)$ that $(V, v)$ is a valued $K$-vector space. Then $(V, v)$ is also a valued $F$-vector space. Similarly, if ${ }_{K} B(V, \gamma)$ is the component of ${ }_{K} V$ corresponding to $\gamma \in v(V)$, then ${ }_{F} B(V, \gamma)$ is the component of ${ }_{F} V$ corresponding to this same $\gamma$. We say that $U$ is valuation disjoint from $K$ in $V$ over $W$ if every subset $X \subset U$ which is $F$-valuation independent in $U$ over $W$, remains $K$ valuation independent in $V$ over $\langle W\rangle$ (it suffices to check this condition for finite $X)$. Note that $U$ is valuation disjoint from $K$ in $V$ over $W$ if and only if $U$ is valuation disjoint from $K$ in $\langle U\rangle$ over $W$. We say that $U$ is valuation disjoint from $K$ in $V$ if $U$ is valuation disjoint from $K$ in $V$ over $W=0$.

Proposition 3.6 $U$ is valuation disjoint from $K$ in $V$ over $W$ if and only if for all $\gamma \in v(U)$, every (finite) $X \subset B(U, \gamma)$ which is $F$-independent over $B(W, \gamma)$, remains $K$-independent in $B(V, \gamma)$ over $B(\langle W\rangle, \gamma)$.

Proof: $\quad$ For $\mathcal{B} \subset U$ and $\gamma \in v(U)$, the set $\pi^{U}(\gamma, \mathcal{B})$ can be canonically identified with $\pi^{V}(\gamma, \mathcal{B})$; for $\gamma \in v(V) \backslash v(U)$, we have that $\pi^{U}(\gamma, \mathcal{B})=\pi^{V}(\gamma, \mathcal{B})=0$. Thus, our assertion follows immediately from Proposition 2.1 .

Note that in general, $\langle B(W, \gamma)\rangle \neq B(\langle W\rangle, \gamma)$. But it is easily shown that

$$
\langle B(W, \gamma)\rangle \subset B(\langle W\rangle, \gamma)
$$

If we add a suitable condition on $W$, then we obtain equality, and the assertion of our proposition can be improved (see Proposition 3.12 below).

Proposition 3.7 Suppose that $U$ admits an F-valuation basis over $W$. Then the following conditions are equivalent:

1) $U$ is valuation disjoint from $K$ in $V$ over $W$

2) every $F$-valuation basis of $U$ over $W$ remains a $K$-valuation basis of $\langle U\rangle$ over $\langle W\rangle$

3) there exists an $F$-valuation basis of $U$ over $W$ which remains $K$-valuation independent in $V$ over $\langle W\rangle$.

Proof: The implications 1$) \Rightarrow 2$ ) and 2$) \Rightarrow 3$ ) are easy to establish. Let us prove $3) \Rightarrow 1)$. By Proposition 3.6 it suffices to show that for all $\gamma \in v(V)$, every finite $X \subset$ $B(U, \gamma)$ which is $F$-independent over $B(W, \gamma)$, remains $K$-independent in $B(V, \gamma)$ over $B(\langle W\rangle, \gamma)$. By Proposition 3.2, where $W^{\prime}=B(\langle W\rangle, \gamma)$, it suffices to show that there exists an $F$-basis of $B(U, \gamma)$ over $B(W, \gamma)$ which remains $K$-independent 
in $B(V, \gamma)$ over $B(\langle W\rangle, \gamma)$. Let $\mathcal{B}$ be the valuation basis of ${ }_{F} U$ over $W$ which remains $K$-valuation independent in $V$ over $\langle W\rangle$. By Proposition $2.1, \pi^{U}(\mathcal{B}, \gamma)$ is a basis of $B(U, \gamma)$ over $B(W, \gamma)$. Since $\mathcal{B}$ remains $K$-valuation independent in $V$ over $\langle W\rangle$, the same proposition shows that $\pi^{U}(\mathcal{B}, \gamma)$ (which is canonically identified with $\pi^{V}(\mathcal{B}, \gamma)$ ) remains $K$-independent in $B(V, \gamma)$ over $B(\langle W\rangle, \gamma)$.

Corollary 3.8 Suppose that $U$ is valuation disjoint from $K$ in $V$ over $W$ and that $W$ is nice in $U$. Then $U$ is disjoint from $K$ in $V$ over $W$.

Proof: $\quad$ Let $X \subset U$ be finite and $F$-independent in $U$ over $W$. Since $W$ is nice in $U$, it is also nice in the subspace ${ }_{F}\langle X\rangle+W$ of $U$. Hence by Theorem 2.2, there exists a valuation basis $\mathcal{B}$ of this subspace over $W$. Since $U$ is valuation disjoint from $K$ in $V$ over $W$, Proposition 3.7 shows that $\mathcal{B}$ remains a $K$-valuation basis of ${ }_{K}\langle\mathcal{B}\rangle+\langle W\rangle$ over $\langle W\rangle$. Hence $\mathcal{B}$ and thus also $X$ is $K$-independent in $V$ over $\langle W\rangle$.

We have the following analogue to Proposition 3.4:

Proposition 3.9 (Transitivity)

Suppose that ${ }_{F} U \subset{ }_{K}{ }^{\prime} V^{\prime} \subset{ }_{K} V$. If $U$ is valuation disjoint from $K^{\prime}$ in $V^{\prime}$ over $W$ and $V^{\prime}$ is valuation disjoint from $K$ in $V$ over ${ }_{K^{\prime}}\langle W\rangle$, then $U$ is valuation disjoint from $K$ in $V$ over $W$. Conversely, if $U$ is valuation disjoint from $K$ in $V$ over $W$, then $U$ is valuation disjoint from $K^{\prime}$ in $V^{\prime}$ over $W$; if in addition $V^{\prime}={ }_{K^{\prime}}\langle U\rangle$ and $W=0$, then $V^{\prime}$ is valuation disjoint from $K$ in $V$.

Proof: As in Proposition 3.4, the first assertion follows from $\langle W\rangle={ }_{K}\left\langle{ }_{K^{\prime}}\langle W\rangle\right\rangle$.

For the converse, let $X \subset U$ be $F$-valuation independent over $W$. By hypothesis, it remains $K$-valuation independent in ${ }_{K} V$ over $\langle W\rangle$. Hence it is also $K^{\prime}$-valuation independent in ${ }_{K^{\prime}} V^{\prime}$ over ${ }_{K^{\prime}}\langle W\rangle \subset\langle W\rangle$.

Assume that $U$ is valuation disjoint from $K$ in $V$. We show that ${ }_{K^{\prime}}\langle U\rangle$ is valuation disjoint from $K$ in $V$. Let $Y \subset{ }_{K^{\prime}}\langle U\rangle$ be a finite $K^{\prime}$-valuation independent set. There exists a finite $F$-independent set $X \subset U$ such that

$$
{ }_{K^{\prime}}\langle Y\rangle \subset{ }_{K^{\prime}}\langle X\rangle \text {. }
$$

By hypothesis, ${ }_{F}\langle X\rangle$ is valuation disjoint from $K$ in $V$, and by Theorem 2.2, there exists an $F$-valuation basis $\mathcal{B}$ of ${ }_{F}\langle X\rangle$. This basis remains a $K$-valuation basis of ${ }_{K}\langle X\rangle$ by Proposition 3.7. Moreover, $\mathcal{B}$ is a system of generators of ${ }_{K^{\prime}}\langle X\rangle$. Hence, $\mathcal{B}$ is a $K^{\prime}$-valuation basis of ${ }_{K^{\prime}}\langle X\rangle$ which remains a $K$-valuation basis of ${ }_{K}\langle X\rangle$. It follows by Proposition 3.7 that ${ }_{K^{\prime}}\langle X\rangle$ is valuation disjoint from $K$ in $V$. Hence, $Y$ is $K$-valuation independent in $V$.

Proposition 3.10 Suppose that $U$ is valuation disjoint from $K$ in $V$. Then

$$
v(U)=v(\langle U\rangle)
$$

Further, $\left\langle U^{\gamma}\right\rangle=\langle U\rangle^{\gamma}$ and $\left\langle U_{\gamma}\right\rangle=\langle U\rangle_{\gamma}$, and for all $\gamma \in v(V)$,

$$
\langle B(U, \gamma)\rangle=B(\langle U\rangle, \gamma) \text {. }
$$


Proof: Assume that $U$ is valuation disjoint from $K$ in $V$. To prove that $v(U)=$ $v(\langle U\rangle)$, it suffices to prove the inclusion " $\supset$ " since the reverse is trivial. So we take $y \in\langle U\rangle$. Then there is a finite-dimensional $F$-subspace $U_{0} \subset U$ such that $y \in\left\langle U_{0}\right\rangle$. By Theorem 2.2, there exists an $F$-valuation basis $\mathcal{B}$ of $U_{0}$. By hypothesis, $\mathcal{B}$ remains a valuation basis of ${ }_{K}\left\langle U_{0}\right\rangle$. Let $y=\sum_{x \in \mathcal{B}} k_{x} x$ with $k_{x} \in K$; then

$$
v(y)=\min _{\left\{x \in \mathcal{B} ; k_{x} \neq 0\right\}} v(x) \in v\left(U_{0}\right) \subset v(U),
$$

as required. We see that $v(x) \geq v(y)$ whenever $k_{x} \neq 0$. Consequently, $\langle U\rangle^{\gamma} \subset\left\langle U^{\gamma}\right\rangle$ and $\langle U\rangle_{\gamma} \subset\left\langle U_{\gamma}\right\rangle$. Since the reverse inclusions follow from the ultrametric triangle law, we obtain that equality holds. Further, with $\gamma=v(y)$,

$$
\pi^{V}(y, \gamma)=\pi^{V}\left(\sum_{x \in \mathcal{B}} k_{x} x, \gamma\right)=\sum_{x \in \mathcal{B}} k_{x} \pi^{U}(x, \gamma) \in\langle B(U, \gamma)\rangle
$$

From this, it follows that $B(\langle U\rangle, \gamma) \subset\langle B(U, \gamma)\rangle$. Together with the reverse inclusion that we have already mentioned, this yields the desired equality.

Remark 3.11 In the course of the foregoing proof, we have shown the following fact: Assume that $U$ is valuation disjoint from $K$ in $V$. Then for all $y \in\langle U\rangle$, there exists a finite set $X \subset U, K$-valuation independent in $V$, and $k_{x} \in K$ such that

$$
y=\sum_{x \in X} k_{x} x
$$

Proposition 3.12 Suppose that $W$ is valuation disjoint from $K$ in $V$. Then $U$ is valuation disjoint from $K$ in $V$ over $W$ if and only if $B(U, \gamma)$ is disjoint from $K$ in $B(V, \gamma)$ over $B(W, \gamma)$, for all $\gamma \in v(U)$.

Proof: $\quad$ By Proposition 3.10, $\langle B(W, \gamma)\rangle=B(\langle W\rangle, \gamma)$; so the assertion follows from Proposition 3.6.

Corollary 3.13 Suppose that $W$ is valuation disjoint from $K$ in $V$. If $U$ is valuation disjoint from $K$ in $V$ over $W$, then $U$ is valuation disjoint from $K$ in $V$.

Proof: By Proposition 3.12, it suffices to show that $B(U, \gamma)$ is disjoint from $K$ in $B(V, \gamma)$, for all $\gamma \in v(U)$. But by hypothesis and Proposition 3.12, we have that $B(W, \gamma)$ is disjoint from $K$ in $B(V, \gamma)$ and $B(U, \gamma)$ is disjoint from $K$ in $B(V, \gamma)$ over $B(W, \gamma)$. The desired result now follows from Proposition 3.5.

\subsection{Correct extensions}

We say that ${ }_{F} U \subset{ }_{K} V$ is a correct extension if $U$ is valuation disjoint from $K$ in $V$ and $\langle U\rangle$ is nice in $V$. Note that ${ }_{F} 0 \subset{ }_{K} V$ is a correct extension.

Lemma 3.14 If $U$ is valuation disjoint from $K$ in $V$, then $U$ is nice in $\langle U\rangle$ as a valued $F$-subspace. 
Proof: Take $y \in\langle U\rangle \backslash U$; we have to show that the extension $U \subset U+F y$ admits a valuation basis. As in Remark 3.11, let $X \subset U$ be $K$-valuation independent in $V$ and $k_{x} \in K \backslash\{0\}$ such that $y=\sum_{x \in X} k_{x} x$. Then $k_{x} \notin F$ for at least one $x \in X$. After subtraction of a suitable element from $U$, we can even assume that all $k_{x} \neq 0$ are not in $K$. Set $\gamma=v(y)$. Then $\gamma=v(x)$ for some $x \in X$ such that $k_{x} \notin K$. By Proposition 2.1, $\pi^{V}(\gamma, X)$ is $K$-independent in $B(V, \gamma)$. So by Remark 3.1,

$$
\pi^{V}(\gamma, y)=\sum_{x \in X} k_{x} \pi^{V}(\gamma, x) \notin B(U, \gamma)
$$

hence $\{y\}$ is a valuation basis of $U+F y$ over $U$ (again by Proposition 2.1).

Suppose ${ }_{K} U \subset{ }_{K} V$. Then ${ }_{K} U$ is nice in ${ }_{K} V$ if and only if ${ }_{F} U$ is nice in ${ }_{F} V$. Indeed, we see from the definition that niceness does not depend on the base field. So we get the following:

Corollary 3.15 Suppose ${ }_{F} U \subset{ }_{K} V$ is a correct extension. Then $\langle U\rangle$ and $U$ are nice in $V$ as valued $F$-subspaces.

Proof: By hypothesis and Lemma 3.14, $U$ is nice in $\langle U\rangle$ as a valued $F$-subspace. But by hypothesis, $\langle U\rangle$ is nice in $V$. So the assertion follows by transitivity of niceness (cf. $[\mathrm{F}]$ or $[\mathrm{KF}]$ ).

Proposition 3.16 Suppose that ${ }_{F} U \subset{ }_{K^{\prime}} V^{\prime} \subset{ }_{K} V$ such that ${ }_{F} U \subset{ }_{K} V$ is a correct extension. Then ${ }_{F} U \subset{ }_{K^{\prime}} V^{\prime}$ is also a correct extension.

Proof: We have that $U$ is valuation disjoint from $K$ in $V$ and $\langle U\rangle$ is nice in $V$. By Proposition 3.9, $U$ is valuation disjoint from $K^{\prime}$ in $V^{\prime}$. It remains to show that ${ }_{K^{\prime}}\langle U\rangle$ is nice in $V^{\prime}$. We will even show that ${ }_{K^{\prime}}\langle U\rangle$ is nice in $V$. First we observe that ${ }_{K^{\prime}}\langle U\rangle \subset{ }_{K} V$ is a correct extension: by hypothesis, $\langle U\rangle={ }_{K}\left\langle{ }_{K^{\prime}}\langle U\rangle\right\rangle$ is nice in $V$; and by Proposition $3.9,{ }_{K}{ }^{\prime}\langle U\rangle$ is valuation disjoint from $K$ in $V$. Now by Corollary $3.15,{ }_{K^{\prime}}\langle U\rangle$ is nice in $V$.

Lemma 3.17 Let ${ }_{F} U \subset{ }_{K} V$ be a correct extension and ${ }_{F} U \subset{ }_{K} V^{\prime} \subset{ }_{K} V$. Suppose that $V^{\prime}$ admits a finite $K^{\prime}$-valuation basis over ${ }_{K^{\prime}}\langle U\rangle$ which remains $K$-valuation independent over ${ }_{K}\langle U\rangle$. Then ${ }_{K}{ }^{\prime} V^{\prime} \subset{ }_{K} V$ is also a correct extension.

Proof: By Lemma 3.7, $V^{\prime}$ is valuation disjoint from $K$ in $V$ over ${ }_{K^{\prime}}\langle U\rangle$, and by Proposition $3.9,{ }_{K^{\prime}}\langle U\rangle$ is valuation disjoint from $K$ in $V$. Hence by Corollary 3.13 , $V^{\prime}$ is valuation disjoint from $K$ in $V$. Moreover, ${ }_{K}\left\langle V^{\prime}\right\rangle$ is nice in $V$ : since by hypothesis $\langle U\rangle$ is nice in $V$ and $\langle U\rangle \subset{ }_{K}\left\langle V^{\prime}\right\rangle$ is finite, the assertion follows from Theorem 2.2.

We say that ${ }_{K} V$ is finitely generated over ${ }_{F} U$ if ${ }_{F} U \subset{ }_{K} V, K$ is finitely generated over $F$ as a field, and $\operatorname{dim}_{K}(V /\langle U\rangle)<\infty$. If ${ }_{F} U \subset{ }_{K^{\prime}} V^{\prime} \subset{ }_{K} V$ and ${ }_{K^{\prime}} V^{\prime}$ is finitely generated over ${ }_{F} U$, then $\left\langle V^{\prime}\right\rangle$ is finitely generated over $\langle U\rangle$. 
Proposition 3.18 Let ${ }_{F} U \subset{ }_{K} V$ be a correct extension and ${ }_{K^{\prime}} V^{\prime} \subset{ }_{K} V$ such that ${ }_{K}{ }^{\prime} V^{\prime}$ is finitely generated over ${ }_{F} U$. Then there exists $K^{\prime \prime} V^{\prime \prime}$, finitely generated over ${ }_{F} U$, such that ${ }_{K^{\prime}} V^{\prime} \subset{ }_{K^{\prime \prime}} V^{\prime \prime} \subset{ }_{K} V$ and ${ }_{K}{ }^{\prime \prime} V^{\prime \prime} \subset{ }_{K} V$ is a correct extension.

Proof: We wish to show that there exists $K^{\prime \prime}$ finitely generated over $K^{\prime}$ and a vector space $K^{\prime \prime} V^{\prime \prime}$, finitely generated over ${ }_{K}{ }^{\prime \prime}\left\langle V^{\prime}\right\rangle$, such that $V^{\prime \prime}$ admits a $K^{\prime \prime}$ valuation basis over ${ }^{\prime \prime}\langle U\rangle$ which remains $K$-valuation independent in $V$ over $\langle U\rangle$. Then $K^{\prime \prime} V^{\prime \prime}$ will be the required extension: clearly, $K^{\prime \prime} V^{\prime \prime}$ is finitely generated over ${ }_{F} U$, and the last assertion follows from Lemma 3.17. We have that ${ }_{K^{\prime}}\langle U\rangle \subset{ }_{K^{\prime}} V^{\prime}$ is a finite extension; so let $\left\{x_{1}, \ldots, x_{n}\right\}$ be a basis of ${ }_{K^{\prime}} V^{\prime}$ over ${ }_{K^{\prime}}\langle U\rangle$. The extension $\langle U\rangle \subset\left\langle V^{\prime}\right\rangle$ is also finite. Since $\langle U\rangle$ is nice in $V$ and thus also in $\left\langle V^{\prime}\right\rangle$ by hypothesis, Theorem 2.2 shows that $\left\langle V^{\prime}\right\rangle$ admits a $K$-valuation basis $\mathcal{B}$ over $\langle U\rangle$. Let $K^{\prime \prime}$ be a finitely generated extension of $K^{\prime}$ such that every $x_{i}$ is a $K^{\prime \prime}$-linear combination of elements of $\mathcal{B}$ and of $U$. Set

$$
V^{\prime \prime}:={ }_{K^{\prime \prime}}\langle\mathcal{B} \cup U\rangle
$$

Then $\mathcal{B}$ is a $K^{\prime \prime}$-valuation basis of $V^{\prime \prime}$ over ${ }_{K}^{\prime \prime}\langle U\rangle$ which remains $K$-valuation independent in $V$ over $\langle U\rangle$.

Lemma 3.19 Suppose ${ }_{F} U \subset{ }_{K} V$ and ${ }_{F} U$ is valuation disjoint from $K$ in ${ }_{K} V$. Then there exists ${ }_{K} V^{\prime} \subset{ }_{K} V$ such that ${ }_{F} U \subset{ }_{K} V^{\prime}$ is a correct extension and ${ }_{K} V^{\prime} \subset{ }_{K} V$ is an immediate extension.

Proof: By Theorem 2.3, there exists ${ }_{K} V^{\prime}$ with $\langle U\rangle \subset{ }_{K} V^{\prime} \subset{ }_{K} V$ such that $\langle U\rangle$ is nice in $V^{\prime}$ and ${ }_{K} V^{\prime} \subset_{K} V$ is an immediate extension. Since $U$ is valuation disjoint from $K$ in $V^{\prime}$, we have that ${ }_{F} U \subset{ }_{K} V^{\prime}$ is a correct extension.

\section{Lifting embeddings of skeletons}

Let ${ }_{K} V$ and ${ }_{K}^{\prime} V^{\prime}$ be vector spaces with common $F$-subspace ${ }_{F} U$. An embedding of vector spaces $h:{ }_{K} V \rightarrow{ }_{K}{ }^{\prime} V^{\prime}$ over $U$ consists of an embedding $\sigma: K \rightarrow K^{\prime}$ of fields and an embedding $\varphi: V \rightarrow V^{\prime}$ of abelian groups with $\left.\varphi\right|_{U}=\mathrm{id}$, such that

$$
\forall k \in K \forall x \in V: \varphi(k x)=\sigma(k) \varphi(x) .
$$

Now let $V$ and $V^{\prime}$ be equipped with valuations $v$ and $v^{\prime}$ respectively, which coincide on $U$. An embedding of skeletons $f_{S}: S\left({ }_{K} V, v\right) \rightarrow S\left(K^{\prime} V^{\prime}, v^{\prime}\right)$ over $S\left({ }_{F} U, v\right)$ consists of a field embedding $\sigma: K \rightarrow K^{\prime}$ with $\left.\sigma\right|_{F}=\mathrm{id}$, an order embedding $\tau: v(V) \rightarrow v^{\prime}\left(V^{\prime}\right)$ with $\left.\tau\right|_{v(U)}=$ id and vector space embeddings $h_{\gamma}:{ }_{K} B(V, \gamma) \rightarrow{ }_{K^{\prime}} B\left(V^{\prime}, \tau(\gamma)\right)$ over $B(U, \gamma)$ for all $\gamma \in v(V)$. Further, an embedding of valued vector spaces $f:\left({ }_{k} V, v\right) \rightarrow\left({ }_{K}{ }^{\prime} V^{\prime}, v^{\prime}\right)$ over $U$ consists of an embedding $(\sigma, \varphi):{ }_{K} V \rightarrow{ }_{K}{ }^{\prime} V^{\prime}$ over $U$ and an embedding $\tau: v(V) \rightarrow v^{\prime}\left(V^{\prime}\right)$ such that for all $x \in V$,

$$
\tau(v(x))=v^{\prime}(\varphi(x)) .
$$


If $K=K^{\prime}$, then we will speak of an embedding of valued $K$-vector spaces if $\sigma=\mathrm{id}$. The so defined embeddings are isomorphisms if all involved maps are surjective.

Every embedding $f=(\sigma, \varphi, \tau)$ of valued vector spaces induces an embedding $f_{S}=\left(\sigma, \tau,\left\{h_{\gamma} ; \gamma \in v(V)\right\}\right)$ of their skeletons, which satisfies:

$$
\forall x \in V: \pi^{V^{\prime}}(\tau(v(x)), \varphi(x))=h_{v(x)}\left(\pi^{V}(v(x), x)\right) .
$$

If $f$ induces $f_{S}$, then we will also say that $f_{S}$ lifts to $f$. In this section, we will consider how embeddings of skeletons can be lifted to embeddings of valued vector spaces. We will first discuss the case of a fixed base field.

Proposition 4.1 Let $(U, v) \subset(V, v)$ and $(U, v) \subset\left(V^{\prime}, v^{\prime}\right)$ be extensions of valued $K$-vector spaces. If $V$ admits a valuation basis over $U$, then every embedding of $S(V, v)$ in $S\left(V^{\prime}, v^{\prime}\right)$ over $S(U, v)$ lifts to an embedding of $(V, v)$ in $\left(V^{\prime}, v^{\prime}\right)$ over $U$.

Proof: $\quad$ Let $\mathcal{B}$ be a valuation basis of $V$ over $U$, and $\left(\operatorname{id}_{K}, \tau,\left\{h_{\gamma} ; \gamma \in v(V)\right\}\right)$ : $S(V, v) \rightarrow S\left(V^{\prime}, v^{\prime}\right)$ an embedding. From Proposition 2.1 we know that $\pi^{V}(\gamma, \mathcal{B})$ is a $K$-basis of $B(V, \gamma)$ over $B(U, \gamma)$, for every $\gamma \in v(V)$. Consequently, the set $h_{\gamma}\left(\pi^{V}(\gamma, \mathcal{B})\right)$ is $K$-independent in $B\left(V^{\prime}, \tau(\gamma)\right)$ over $B(U, \tau(\gamma))$. We choose a set $\mathcal{B}^{\prime}$ of representatives in $V^{\prime}$ for the elements of $\bigcup_{\gamma \in v(V)} h_{\gamma}\left(\pi^{V}(\gamma, \mathcal{B})\right)$. By Proposition $2.1, \mathcal{B}^{\prime}$ is $K^{\prime}$-valuation independent in $V^{\prime}$ over $U$. Hence, every injective map from $\mathcal{B}$ into $\mathcal{B}^{\prime}$ extends additively to an embedding $\varphi$ of $V$ into $V^{\prime}$ over $U$ as $K$ vector spaces. We choose this map as follows: for each $x \in \mathcal{B}$ we let its image in $\mathcal{B}^{\prime}$ be the representant of $h_{v(x)}\left(\pi^{V}(v(x), x)\right)$.

To show that $\varphi$ has the required properties, let $x \in V$ and write $x=\sum_{i=1}^{n} k_{i} x_{i}$ with $x_{i} \in \mathcal{B}$ and $0 \neq k_{i} \in K$. Since $\mathcal{B}$ is a valuation basis, we have that $v\left(x_{i}\right) \geq v(x)$ for all $i$, and by virtue of the ultrametric triangle law, there is at least one $i_{0}$ such that $v\left(x_{i_{0}}\right)=v(x)$. If $v\left(x_{i}\right)>v\left(x_{i_{0}}\right)$ then also $v^{\prime}\left(\varphi\left(x_{i}\right)\right)=\tau v\left(x_{i}\right)>\tau v\left(x_{i_{0}}\right)=$ $v^{\prime}\left(\varphi\left(x_{i_{0}}\right)\right)$ since $\tau$ is an order isomorphism. Since $\mathcal{B}^{\prime}=\varphi(\mathcal{B})$ is a valuation basis, we have that

$$
v^{\prime}(\varphi(x))=v^{\prime}\left(\sum_{i=1}^{n} k_{i} \varphi\left(x_{i}\right)\right)=\min _{i} v^{\prime}\left(\varphi\left(x_{i}\right)\right)=v^{\prime}\left(\varphi\left(x_{i_{0}}\right)\right)=\tau\left(v\left(x_{i_{0}}\right)\right)=\tau(v(x)) .
$$

This proves that $\varphi$ is an embedding of valued vector spaces. To prove that $\left(\operatorname{id}_{K}, \varphi, \tau\right)$ lifts $\left(\operatorname{id}_{K}, \tau,\left\{h_{\gamma} ; \gamma \in v(V)\right\}\right)$, it remains to show that $\varphi$ induces the embeddings $h_{\gamma}$ on the components. If $v\left(x_{i}\right)=v(x)$, then we have that

$$
\pi^{V^{\prime}}\left(\tau(v(x)), \varphi\left(x_{i}\right)\right)=h_{v(x)}\left(\pi^{V}\left(v(x), x_{i}\right)\right)
$$

by our construction of $\varphi$. If on the other hand $v\left(x_{i}\right)>v(x)$, then

$$
\pi^{V^{\prime}}\left(\tau(v(x)), \varphi\left(x_{i}\right)\right)=0=h_{v(x)}(0)=h_{v(x)}\left(\pi^{V}\left(v(x), x_{i}\right)\right) .
$$

So we have that

$$
\begin{aligned}
\pi^{V^{\prime}}(\tau(v(x)), \varphi(x)) & =\pi^{V^{\prime}}\left(\tau(v(x)), \sum_{i=1}^{n} k_{i} \varphi\left(x_{i}\right)\right)=\sum_{i=1}^{n} k_{i} \pi^{V^{\prime}}\left(\tau(v(x)), \varphi\left(x_{i}\right)\right) \\
& =\sum_{i=1}^{n} k_{i} h_{v(x)}\left(\pi^{V}\left(v(x), x_{i}\right)\right)=h_{v(x)}\left(\pi^{V}\left(v(x), \sum_{i=1}^{n} k_{i} x_{i}\right)\right) \\
& =h_{v(x)}\left(\pi^{V}(v(x), x)\right),
\end{aligned}
$$


as required.

Corollary 4.2 Let $(U, v) \subset(V, v)$ and $(U, v) \subset\left(V^{\prime}, v^{\prime}\right)$ be extensions of valued $K$-vector spaces. Then for every isomorphism $f_{S}: S(V, v) \simeq S\left(V^{\prime}, v^{\prime}\right)$ over $S(U, v)$ there are valued $K$-subspaces $\left(V_{0}, v\right) \subset(V, v)$ and $\left(V_{0}^{\prime}, v^{\prime}\right) \subset\left(V^{\prime}, v^{\prime}\right)$ such that:

1) $f_{S}$ lifts to an isomorphism $f:\left(V_{0}, v\right) \simeq\left(V_{0}^{\prime}, v^{\prime}\right)$ over $U$

2) the extensions $\left(V_{0}, v\right) \subset(V, v)$ and $\left(V_{0}^{\prime}, v^{\prime}\right) \subset\left(V^{\prime}, v^{\prime}\right)$ are immediate.

Proof: We take $\mathcal{B}$ to be a maximal valuation independent set in $V$ over $U$ and $V_{0}$ the subspace of $V$ generated by $\mathcal{B}$ over $U$. Then we take $\mathcal{B}^{\prime}$ as in the foregoing proof, and let $V_{0}^{\prime}$ be the subspace of $V^{\prime}$ generated by $\mathcal{B}^{\prime}$ over $U$. Theorem 2.3 shows that assertion 2) holds. Hence, $S\left(V_{0}, v\right)=S(V, v) \simeq S\left(V^{\prime}, v^{\prime}\right)=S\left(V_{0}^{\prime}, v^{\prime}\right)$ over $S(U, v)$. Now the foregoing proposition gives the desired isomorphism.

We turn to the case of a variable base field. For the remainder of this section, we assume that

$$
\left({ }_{F} U, v\right) \subset\left({ }_{K} V, v\right) \text { and } \quad\left({ }_{F} U, v\right) \subset\left({ }_{K}{ }^{\prime} V^{\prime}, v^{\prime}\right)
$$

are extensions of valued vector spaces. By ${ }_{K}^{V}\langle U\rangle$ and ${ }_{K^{\prime}}^{V^{\prime}}\langle U\rangle$ we denote the subspaces generated by $U$ in ${ }_{K} V$ and in ${ }_{K^{\prime}} V^{\prime}$ respectively.

Proposition 4.3 Suppose that $U$ is valuation disjoint from $K$ in $V$ and from $K^{\prime}$ in $V^{\prime}$. Let $\sigma: K \rightarrow K^{\prime}$ be a field embedding over $F$, and $\mathcal{B}$ an $F$-basis of $U$. If

$$
\varphi:{ }_{K}^{V}\langle U\rangle \longrightarrow{ }_{\sigma(K)}^{V^{\prime}}\langle U\rangle
$$

is defined by

$$
\forall b_{1}, \ldots, b_{n} \in \mathcal{B} \forall k_{1}, \ldots, k_{n} \in K: \varphi\left(\sum_{i}^{n} k_{i} b_{i}\right):=\sum_{i}^{n} \sigma\left(k_{i}\right) b_{i},
$$

then $\tilde{f}=\left(\sigma, \varphi, i d_{v(U)}\right):\left({ }_{K}^{V}\langle U\rangle, v\right) \simeq\left({ }_{\sigma(K)}^{V^{\prime}}\langle U\rangle, v^{\prime}\right)$ is an isomorphism of valued vector spaces over $U$. It is uniquely determined by $\sigma$.

Proof: $\quad$ Since $U$ is valuation disjoint from $K$ in $V$, it follows from Corollary 3.8 (with $W=0$ ) that $U$ is disjoint from $K$ in $V$. Hence by Corollary 3.3, $\mathcal{B}$ remains a $K$-basis of ${ }_{K}^{V}\langle U\rangle$. Similarly, $\mathcal{B}$ remains a $K^{\prime}$-basis of ${ }_{K^{\prime}}^{\gamma^{\prime}}\langle U\rangle$. Hence, $\varphi$ is well defined and bijective, and $(\sigma, \varphi)$ is a vector space isomorphism. Moreover, it is uniquely determined by $\sigma$.

From Proposition 3.10 we know that $v\left({ }_{K}^{V}\langle U\rangle\right)=v(U)=v^{\prime}\left({ }_{K^{\prime}}^{V^{\prime}}\langle U\rangle\right)$. Hence it suffices to show that $v^{\prime}(\varphi(y))=v(y)$ for all $y \in{ }_{K}^{V}\langle U\rangle$. As in Remark 3.11, let $X \subset U$ be $K$-valuation independent in $V$, and $k_{x} \in K$ such that $y=\sum_{x \in X} k_{x} x$. Then $X$ is $F$-valuation independent in $U$, and it remains $K^{\prime}$-valuation independent 
in $V^{\prime}$ because $U$ is valuation disjoint from $K^{\prime}$ in $V^{\prime}$. Further, $k_{x}=0$ if and only if $\sigma\left(k_{x}\right)=0$. So we have that

$$
\begin{aligned}
v^{\prime}(\varphi(y)) & =v^{\prime}\left(\sum_{x \in X} \sigma\left(k_{x}\right) \varphi(x)\right)=v^{\prime}\left(\sum_{x \in X} \sigma\left(k_{x}\right) x\right)=\min _{\left\{x \in X ; k_{x} \neq 0\right\}} v^{\prime}(x) \\
& =\min _{\left\{x \in X ; k_{x} \neq 0\right\}} v(x)=v\left(\sum_{x \in X} k_{x} x\right)=v(y) .
\end{aligned}
$$

Proposition 4.4 Let $f_{S}=\left(\sigma, \tau,\left\{h_{\gamma} ; \gamma \in v(V)\right\}\right): S\left({ }_{K} V, v\right) \rightarrow S\left({ }_{K}{ }^{\prime} V^{\prime}, v^{\prime}\right)$ be an embedding over $S\left({ }_{F} U, v\right)$. If $U$ is valuation disjoint from $K$ in $V$, then $U$ is also valuation disjoint from $\sigma(K)$ in $V^{\prime}$.

Proof: $\quad$ Since $U$ is valuation disjoint from $K$ in $V$, Proposition 3.12 (with $W=0$ ) shows that $B(U, \gamma)$ is disjoint from $K$ in $B(V, \gamma)$, for all $\gamma \in v(U)$. Since $\tau(\gamma)=\gamma$ for all $\gamma \in v(U)$, we find that for those $\gamma, h_{\gamma}$ is an embedding from ${ }_{K} B(V, \gamma)$ onto a $\sigma(K)$-subspace of $B\left(V^{\prime}, \gamma\right)$ over $B(U, \gamma)$. Hence by Remark 3.1, $B(U, \gamma)$ is disjoint from $\sigma(K)$ in $B\left(V^{\prime}, \gamma\right)$, for all $\gamma \in v(U)$. Again by Proposition 3.12, we obtain that $U$ is valuation disjoint from $\sigma(K)$ in $V^{\prime}$.

Corollary 4.5 Assume that $U$ is valuation disjoint from $K$ in $V$. Then for every embedding $f_{S}: S\left({ }_{K} V, v\right) \rightarrow S\left({ }_{K^{\prime}} V^{\prime}, v^{\prime}\right)$ over $S\left({ }_{F} U, v\right)$, there exists an isomorphism $\tilde{f}:\left({ }_{K}^{V}\langle U\rangle, v\right) \simeq\left({ }_{\sigma(K)}^{V^{\prime}}\langle U\rangle, v^{\prime}\right)$ over $U$ such that $f_{S}$ extends the skeleton isomorphism $\tilde{f}_{S}$ induced by $\tilde{f}$. (We may view $\tilde{f}$ as an embedding of $(\underset{K}{V}\langle U\rangle, v)$ in $\left.\left(\begin{array}{l}V_{K}^{\prime} \\ K^{\prime}\end{array} U\right\rangle, v^{\prime}\right)$.)

Proof: From the foregoing proposition, we know that $U$ is also valuation disjoint from $\sigma(K)$ in $V^{\prime}$. Thus, from Proposition 4.3 we obtain an isomorphism $\tilde{f}:\left({ }_{K}^{V}\langle U\rangle, v\right) \simeq\left({ }_{\sigma(K)}^{V^{\prime}}\langle U\rangle, v^{\prime}\right)$ over $U$. To prove that $f_{S}$ extends $\tilde{f}_{S}$ it suffices to show that $\tilde{f}$ induces the corresponding restrictions of the maps $h_{\gamma}$ on the components $B\left({ }_{K}^{V}\langle U\rangle, \gamma\right)$. From Proposition 3.10 we infer that $B\left({ }_{K}^{V}\langle U\rangle, \gamma\right)={ }_{K}\langle B(U, \gamma)\rangle$. But the embeddings on this space over $B(U, \gamma)$ are uniquely determined by $\sigma: K \rightarrow K^{\prime}$, since for every linear combination $k_{1} x_{1}+\ldots+k_{n} x_{n}$ with $x_{i} \in B(U, \gamma)$ and $k_{i} \in K$, the image must be $\sigma\left(k_{1}\right) x_{1}+\ldots+\sigma\left(k_{n}\right) x_{n}$.

Corollary 4.6 Let $f_{S}: S\left({ }_{K} V, v\right) \rightarrow S\left({ }_{K}, V^{\prime}, v^{\prime}\right)$ be an embedding over $S\left({ }_{F} U, v\right)$. Assume that $\left({ }_{F} U, v\right) \subset\left({ }_{K_{1}} V_{1}, v\right)$ is a correct subextension of $\left({ }_{F} U, v\right) \subset\left({ }_{K} V, v\right)$ such that $\operatorname{dim}_{K_{1}} V_{1} / V_{K_{1}}\langle U\rangle<\infty$. Then the corresponding restriction of $f_{S}$ lifts to an embedding of $\left(K_{1} V_{1}, v\right)$ in $\left(K^{\prime} V^{\prime}, v^{\prime}\right)$ over $U$. In particular, this holds for finitely generated correct subextensions.

Proof: Set $K_{1}^{\prime}=\sigma\left(K_{1}\right)$. From the foregoing corollary, we obtain an isomorphism $\left.\tilde{f}:\left(\begin{array}{l}V \\ K_{1}\end{array}\langle U\rangle, v\right) \simeq\left(\begin{array}{l}V_{K_{1}^{\prime}}^{\prime} \\ \langle U\end{array}\right\rangle, v^{\prime}\right)$ over $U$. Via this isomorphism, we identify both spaces, and we identify $K_{1}$ with $K_{1}^{\prime}$. Now we may view both $V$ and $V^{\prime}$ as $K_{1}$-vector 
spaces. Since $\operatorname{dim}_{K} V_{1} / K_{K_{1}}\langle U\rangle<\infty$, we know from Theorem 2.2 that $V_{1}$ admits a $K_{1}$-valuation basis over $K_{1}\langle U\rangle$. Hence, Proposition 4.1 shows that the restriction of $f_{S}$ to the skeleton of $\left({ }_{K_{1}}^{V_{1}}\langle U\rangle, v\right)$ lifts to an embedding of $\left(K_{1} V_{1}, v\right)$ in $\left(K_{1} V^{\prime}, v^{\prime}\right)$ over $V_{K_{1}}^{V_{1}}\langle U\rangle$. This is the required embedding of $\left({ }_{K_{1}} V_{1}, v\right)$ in $\left({ }_{K^{\prime}} V^{\prime}, v^{\prime}\right)$ over $U$.

\section{Model theoretic results}

\subsection{Ax-Kochen-Ershov Principles}

In this section, we shall prove Theorem 1.1 and a somewhat more general principle for the elementary equivalence of valued vector spaces over a common subspace (Proposition 5.6). We will use the following general model theoretic principles:

Lemma 5.1 1) Let $\mathcal{M} \subset \mathcal{N}$ and $\mathcal{M} \subset \mathcal{M}^{*}$ be extensions of $\mathcal{L}$-structures. If $\mathcal{N}$ can be embedded over $\mathcal{M}$ in $\mathcal{M}^{*}$ and if $\mathcal{M} \prec \mathcal{M}^{*}$, then $\mathcal{M} \prec \exists \mathcal{N}$. For the converse, assume $\mathcal{M}^{*}$ to be $\kappa$-saturated for some cardinal $\kappa>\operatorname{card}(\mathcal{N})$. If $\mathcal{M} \prec_{\exists} \mathcal{N}$ holds or if every finitely generated subextension $\mathcal{M} \subset \mathcal{N}_{0}$ of $\mathcal{M} \subset \mathcal{N}$ can be embedded over $\mathcal{M}$ in $\mathcal{M}^{*}$, then $\mathcal{N}$ can be embedded over $\mathcal{M}$ in $\mathcal{M}^{*}$. If $\mathcal{M} \prec \mathcal{N}$ holds, then there is even an elementary embedding $\iota$ over $\mathcal{M}$, that is, $\iota(\mathcal{N}) \prec \mathcal{M}^{*}$.

2) If $\operatorname{card}(\mathcal{M})$ is infinite, then for every cardinal $\kappa>\operatorname{card}(\mathcal{M})$ there exists a $\kappa$-saturated elementary extension of $\mathcal{M}$.

3) Isomorphic structures are elementarily equivalent.

4) Assume that $\mathcal{M}_{i}, i \in \mathbb{N}$, are $\mathcal{L}$-structures such that $\mathcal{M}_{i} \prec \mathcal{M}_{i+1}$ for every $i \in \mathbb{N}$, and set $\mathcal{M}_{\omega}:=\bigcup_{i \in \mathbb{N}} \mathcal{M}_{i}$. Then $\mathcal{M}_{i} \prec \mathcal{M}_{\omega}$ for every $i \in \mathbb{N}$.

For details and proofs, cf. e.g. $[\mathrm{C}-\mathrm{K}]$ or $[\mathrm{KF}]$. Further, let us note the following:

a) Formulas in a language of skeletons can be encoded in the corresponding language of valued vector spaces. We refer the reader to [K3]. This yields the "only if"-assertions in Theorem 1.1. We will use this fact without further mention. For the same reason, if $\left({ }_{K} V, v\right)$ is $\kappa$-saturated, then this also holds for $S\left({ }_{K} V, v\right)$.

b) From Proposition 2.1 it follows that $\operatorname{card}\left({ }_{K} V\right)=\max \{\operatorname{card}(K), \operatorname{card}(V)\} \geq$ $\operatorname{card}\left(S\left({ }_{K} V, v\right)\right)$.

Let us first consider immediate extensions.

Proposition 5.2 Let $(U, v) \subset(V, v)$ and $(U, v) \subset\left(V^{*}, v^{*}\right)$ be extensions of valued $K$-vector spaces. Assume that the former extension is immediate and that $\left(V^{*}, v^{*}\right)$ is $\kappa$-saturated, with $\kappa>$ card $(V)$. Then there is an embedding of $(V, v)$ in $\left(V^{*}, v^{*}\right)$ over $U$. Every such embedding induces the identity on $S(V, v)=S(U, v) \subset$ $S\left(V^{*}, v^{*}\right)$.

Proof: By Lemma 5.1, it suffices to show the assertion for $V$ finitely generated over $U$. This can be done by induction on $\operatorname{dim} V / U$; hence we may assume that $\operatorname{dim} V / U=1$. Take $y \in V$ such that $V=U+K y$. Consider the following set of $\mathcal{L}_{\mathrm{V}}^{K}(U \cup\{X\})$-sentences, where $X$ is a new constant symbol:

$$
p:=\{“ v(X+b)=v(c) " ; b, c \in U \text { and } v(y+b)=v(c)\} .
$$


Note that $p$ is nonempty: since $(U, v) \subset(V, v)$ is immediate, we have that $v(y)=$ $v(c)$ for some $c \in U$. We show that $p \cup \operatorname{diagram}(U, v)$ is consistent. So we let $p_{0} \subset p$ be nonempty and finite and show that $p_{0}$ is realisable in $(U, v)$. Consider the following finite subset of $v(U)$ :

$$
\left\{v(c) ; “ v(X+b)=v(c) " \in p_{0}\right\} .
$$

Take $b_{0}, c_{0} \in U$ such that $v\left(c_{0}\right)$ is the maximal element of this set and " $v\left(X+b_{0}\right)=$ $v\left(c_{0}\right)$ " is a corresponding sentence in $p_{0}$. Since $(U, v) \subset(V, v)$ is immediate, there exists $z \in U$ such that $v\left(y+b_{0}+z\right)>v\left(y+b_{0}\right)=v\left(c_{0}\right)$. Set $a=-\left(b_{0}+z\right)$; then for every $b, c \in U$ such that " $v(X+b)=v(c)$ " $\in p_{0}$, we have that $v(a-y)=$ $v(y-a)>v\left(y+b_{0}\right) \geq v(y+b)$, and consequently:

$$
v(a+b)=v(a-y+y+b)=\min \{v(a-y), v(y+b)\}=v(y+b)=v(c) .
$$

This shows that $a$ realises $p_{0}$ in $(U, v)$.

By the saturation property of $\left(V^{*}, v^{*}\right), p$ is realised by some $y^{*} \in V^{*}$. We define $f: V \rightarrow V^{*}$ by:

$$
f(k y+b)=k y^{*}+b \text { for every } k \in K, b \in U
$$

Let us show that

$$
v(k y+b)=v^{*}\left(k y^{*}+b\right)
$$

This is clear if $k=0$; so take $k \neq 0$. Take $c \in U$ such that $v\left(y+k^{-1} b\right)=v(k y+b)=$ $v(c)$. Then " $v\left(X+k^{-1} b\right)=v(c)$ " $\in p$, therefore $v^{*}\left(y^{*}+k^{-1} b\right)=v(c)$ in $\left(V^{*}, v^{*}\right)$. This shows that $v(k y+b)=v(c)=v^{*}\left(k y^{*}+b\right)$, as asserted.

It is clear that $\left.f\right|_{U}=$ id and that $f$ is additive and preserves scalar multiplication. Moreover, $f$ is injective since we have that $k y^{*}+b=0 \Leftrightarrow v^{*}\left(k y^{*}+b\right)=\infty \Leftrightarrow$ $v(k y+b)=\infty \Leftrightarrow k y+b=0$.

It remains to show that for every embedding $f$ of $(V, v)$ in $\left(V^{*}, v^{*}\right)$, the induced skeleton embedding $f_{S}=\left(\mathrm{id}, \tau,\left\{h_{\gamma} ; \gamma \in v(V)\right)\right.$ satisfies that $\tau$ is the identity on $v(V)=v(U) \subset v^{*}\left(V^{*}\right)$ and that $h_{\gamma}$ is the identity on $B(V, \gamma)=B(U, \gamma) \subset B\left(V^{*}, \gamma\right)$ for every $\gamma \in v(V)=v(U)$. The former follows directly from the fact that $f$ is an embedding over $U$. To show the latter, take $x \in V$. Since $(U, v) \subset(V, v)$ is immediate, there is some $z \in U$ such that $v(x-z)>v(x)=v(z)$. Then $v(f(x)-z)=v(f(x-z))=v(x-z)>v(z)$, showing that $\pi(v(x), f(x))=$ $\pi(v(x), z)=\pi(v(x), x)$.

By use of the foregoing proposition and Lemma 5.1, we obtain:

Corollary 5.3 If $(U, v) \subset(V, v)$ is an immediate extension of valued $K$-vector spaces, then $(U, v) \prec_{\exists}(V, v)$.

Combining the foregoing proposition with the results of Section 4, we can prove:

Proposition 5.4 Let $\left({ }_{F} U, v\right) \subset\left({ }_{K} V, v\right)$ and $\left({ }_{F} U, v\right) \subset\left(K_{K^{*}} V^{*}, v^{*}\right)$ be extensions of valued vector spaces. Assume that $U$ is valuation disjoint from $K$ in $V$ and that $\left(K^{*} V^{*}, v^{*}\right)$ is $\kappa$-saturated, with $\kappa>$ card $(V)$. Then every embedding of $S\left({ }_{K} V, v\right)$ in $S\left(K^{*} V^{*}, v^{*}\right)$ over $S\left({ }_{F} U, v\right)$ lifts to an embedding of $\left({ }_{K} V, v\right)$ in $\left(K^{*} V^{*}, v^{*}\right)$ over $U$. 
Proof: First, we embed $\left({ }_{K}^{V}\langle U\rangle, v\right)$ in $\left(K^{*} V^{*}, v^{*}\right)$ over $U$ by use of Proposition 4.3. We identify ${ }_{K}^{V}\langle U\rangle$ with its image in $V^{*}$. Then we take $\mathcal{B} \subset V$ to be maximal $K$-valuation independent over ${ }_{K}^{V}\langle U\rangle$. Afterwards, we embed $\left({ }_{K}^{V}\langle\mathcal{B} \cup U\rangle, v\right)$ in $\left(K^{*} V^{*}, v^{*}\right)$ over ${ }_{K}^{V}\langle U\rangle$ by use of Proposition 4.1. Now the extension $\left({ }_{K}^{V}\langle\mathcal{B} \cup U\rangle, v\right) \subset$ $\left({ }_{K} V, v\right)$ is immediate, and we can apply Proposition 5.2.

Lemma 5.5 Let $\left({ }_{F} U, v\right) \subset\left({ }_{K} V, v\right)$ be an extension of valued vector spaces such that $S\left({ }_{F} U, v\right) \prec_{\exists} S\left({ }_{K} V, v\right)$. Then $U$ is valuation disjoint from $K$ in $V$.

Proof: By Proposition 3.12 (with $W=0$ ), it suffices to show that for every $\gamma \in v(U), B(U, \gamma)$ is disjoint from $K$ in $B(V, \gamma)$. Suppose that $y_{1}, \ldots, y_{n} \in B(U, \gamma)$ are $K$-dependent. Then

$$
\exists z_{1} \ldots \exists z_{n} \in K: \bigvee_{i=1}^{n} z_{i} \neq 0 \wedge \sum_{i=1}^{n} z_{i} y_{i}=0
$$

holds. But this is an existential sentence in the language of skeletons with parameters $\gamma, y_{1}, \ldots, y_{n}$ in $S\left({ }_{F} U, v\right)$ (note that $\gamma$ is needed to talk about the zero in $B(V, \gamma)$, cf. [K3]). It follows by our hypothesis that

$$
\exists z_{1} \ldots \exists z_{n} \in F: \bigvee_{i=1}^{n} z_{i} \neq 0 \wedge \sum_{i=1}^{n} z_{i} y_{i}=0
$$

holds, which says that $y_{1}, \ldots, y_{n}$ are $F$-dependent.

Proposition 5.6 Let $\left({ }_{F} U, v\right) \subset\left({ }_{K} V, v\right)$ and $\left({ }_{F} U, v\right) \subset\left({ }_{K} V^{\prime}, v^{\prime}\right)$ be extensions of valued vector spaces. Assume that $U$ is valuation disjoint from $K$ in $V$. If $S\left({ }_{K} V, v\right) \equiv S\left({ }_{K} V^{\prime}, v^{\prime}\right)$ over $S\left({ }_{F} U, v\right)$, then $\left({ }_{K} V, v\right) \equiv\left(K_{K} V^{\prime}, v^{\prime}\right)$ over $U$.

Proof: The proof proceeds by a model theoretic back and forth construction. We take $\kappa_{1}^{\prime}>\operatorname{card}\left({ }_{K} V\right) \geq \operatorname{card}\left(S\left({ }_{K} V, v\right)\right)$ and choose a $\kappa_{1}^{\prime}$-saturated elementary extension $\left({ }_{K_{1}^{\prime}} V_{1}^{\prime}, v_{1}^{\prime}\right)$ of $\left({ }_{K^{\prime}} V^{\prime}, v^{\prime}\right)$. Then $S\left({ }_{K} V, v\right) \equiv S\left(K^{\prime}, V^{\prime}, v^{\prime}\right) \equiv$ $S\left(K_{1}^{\prime} V_{1}^{\prime}, v_{1}^{\prime}\right)$ over $S\left({ }_{F} U, v\right)$. Since also $S\left(K_{K_{1}^{\prime}} V_{1}^{\prime}, v_{1}^{\prime}\right)$ is $\kappa_{1}^{\prime}$-saturated, we can apply Lemma 5.1 to obtain an elementary embedding $\iota_{1}$ of $S\left({ }_{K} V, v\right)$ in $S\left(K_{1}^{\prime} V_{1}^{\prime}, v_{1}^{\prime}\right)$ over $S\left({ }_{F} U, v\right)$. Since $U$ is assumed to be valuation disjoint from $K$ in $V$, Proposition 5.4 gives an embedding $f_{1}$ of $\left({ }_{K} V, v\right)$ in $\left(K_{1}^{\prime} V_{1}^{\prime}, v_{1}^{\prime}\right)$ over $U$, with $S\left(f_{1}\left(K_{K} V, v\right)\right)=$ $\iota_{1}\left(S\left({ }_{K} V, v\right)\right) \prec S\left(K_{1}^{\prime} V_{1}^{\prime}, v_{1}^{\prime}\right)$. Now we choose $\kappa_{1}>\operatorname{card}\left(K_{K_{1}^{\prime}} V_{1}^{\prime}\right) \geq \operatorname{card}\left(S\left(K_{K_{1}^{\prime}} V_{1}^{\prime}, v_{1}^{\prime}\right)\right)$ and a $\kappa_{1}$-saturated elementary extension $\left(K_{1} V_{1}, v_{1}\right)$ of $\left({ }_{K} V, v\right)$. Then $S\left({ }_{K_{1}^{\prime}} V_{1}^{\prime}, v_{1}^{\prime}\right) \equiv$ $S\left({ }_{K} V, v\right) \equiv S\left(K_{1} V_{1}, v_{1}\right)$ over $S\left({ }_{K} V, v\right) \simeq S\left(f_{1}\left({ }_{K} V, v\right)\right)$. Since also $S\left(K_{1} V_{1}, v_{1}\right)$ is $\kappa_{1}$-saturated, we can apply Lemma 5.1 to obtain an elementary embedding $\iota_{1}^{\prime}$ of $S\left({ }_{K_{1}^{\prime}} V_{1}^{\prime}, v_{1}^{\prime}\right)$ in $S\left(K_{1} V_{1}, v_{1}\right)$ which extends $\iota_{1}^{-1}: S\left(f_{1}\left({ }_{K} V, v\right)\right) \simeq S\left({ }_{K} V, v\right)$. Since $S\left(f_{1}\left(K_{K} V\right), v_{1}^{\prime}\right)=S\left(f_{1}\left({ }_{K} V, v\right)\right) \prec S\left(K_{K_{1}^{\prime}} V_{1}^{\prime}, v_{1}^{\prime}\right)$, we know from Lemma 5.5 that $f_{1}\left({ }_{K} V\right)$ is valuation disjoint from $K_{1}^{\prime}$ in $V_{1}^{\prime}$. Hence we can apply Proposition 5.4 to obtain an embedding $f_{1}^{\prime}$ of $\left(K_{1}^{\prime} V_{1}^{\prime}, v_{1}^{\prime}\right)$ in $\left(K_{1} V_{1}, v_{1}\right)$ which extends the isomorphism $f_{1}^{-1}: f_{1}\left({ }_{K} V, v\right) \simeq\left({ }_{K} V, v\right)$. By induction, we construct elementary extensions $\left(K_{i}^{\prime} V_{i}^{\prime}, v_{i}^{\prime}\right)$ of $\left(K_{K^{\prime}} V^{\prime}, v^{\prime}\right)$ and $\left({ }_{K_{i}} V_{i}, v_{i}\right)$ of $\left({ }_{K} V, v\right)$ together with 
mutual embeddings $f_{i}$ and $f_{i}^{\prime}$. Then we take $\left(K_{\omega}^{\prime} V_{\omega}^{\prime}, v_{\omega}^{\prime}\right),\left(K_{\omega} V_{\omega}, v_{\omega}\right), f_{\omega}$ and $f_{\omega}^{\prime}$ to be the unions over $i \in \mathbb{N}$ of $\left({ }_{K_{i}^{\prime}} V_{i}^{\prime}, v_{i}^{\prime}\right),\left({ }_{K_{i}} V_{i}, v_{i}\right), f_{i}$ and $f_{i}^{\prime}$ respectively. We find that $f_{\omega}:\left(K_{\omega} V_{\omega}, v_{\omega}\right) \rightarrow\left(K_{\omega}^{\prime} V_{\omega}^{\prime}, v_{\omega}^{\prime}\right)$ is an embedding over $U$ with $f_{\omega}^{\prime}$ as its inverse. Hence, $f_{\omega}$ is an isomorphism. Since $\left(K_{i} V_{i}, v_{i}\right) \prec\left(K_{i+1} V_{i+1}, v_{i+1}\right)$ and $\left(K_{i}^{\prime} V_{i}^{\prime}, v_{i}^{\prime}\right) \prec\left(K_{i+1}^{\prime} V_{i+1}^{\prime}, v_{i+1}^{\prime}\right)$ for every $i \in \mathbb{N}$, we can infer from Lemma 5.1 that $\left({ }_{K} V, v\right) \equiv\left({ }_{K_{\omega}} V_{\omega}, v_{\omega}\right) \equiv\left({ }_{K_{\omega}^{\prime}} V_{\omega}^{\prime}, v_{\omega}^{\prime}\right) \equiv\left({ }_{K^{\prime}} V^{\prime}, v^{\prime}\right)$ over $U$.

Now we are ready for the

\section{Proof of Theorem 1.1:}

1) Suppose that $\left({ }_{F} U, v\right) \subset\left({ }_{K} V, v\right)$ such that $S\left({ }_{F} U, v\right) \prec_{\exists} S\left({ }_{K} V, v\right)$. By Lemma 5.5 we know that $U$ is valuation disjoint from $K$ in $V$. We take $\kappa>\operatorname{card}\left({ }_{K} V\right) \geq$ card $\left(S\left({ }_{K} V, v\right)\right)$ and choose a $\kappa$-saturated elementary extension $\left({ }_{F} U, v\right)^{*}$ of $\left({ }_{F} U, v\right)$. Then its skeleton $S\left({ }_{F} U, v\right)^{*}$ is a $\kappa$-saturated elementary extension of $S\left({ }_{F} U, v\right)$. Hence by Lemma 5.1, there is an embedding of $S\left({ }_{K} V, v\right)$ in $S\left({ }_{F} U, v\right)^{*}$ over $S\left({ }_{F} U, v\right)$. We apply Proposition 5.4 to obtain an embedding of $\left({ }_{K} V, v\right)$ in $\left({ }_{F} U, v\right)^{*}$ over $\left({ }_{F} U, v\right)$. In view of Lemma 5.1, this proves that $\left({ }_{F} U, v\right) \prec_{\exists}\left({ }_{K} V, v\right)$.

2) Suppose that $\left({ }_{F} U, v\right) \subset\left({ }_{K} V, v\right)$ is an extension of valued vector spaces such that $S\left({ }_{F} U, v\right) \prec S\left({ }_{K} V, v\right)$. As before, Corollary 5.5 shows that $U$ is valuation disjoint from $K$ in $V$. Since $S\left({ }_{F} U, v\right) \prec S\left({ }_{K} V, v\right)$ means that $S\left({ }_{F} U, v\right)$ and $S\left({ }_{K} V, v\right)$ are elementarily equivalent over $S\left({ }_{F} U, v\right)$, we can infer from Proposition 5.6 that $\left.{ }_{F} U, v\right)$ and $\left({ }_{K} V, v\right)$ are elementarily equivalent over $U$. That is, $\left.{ }_{F} U, v\right) \prec\left({ }_{K} V, v\right)$.

3 ) Assume that $\left({ }_{K} V, v\right)$ and $\left({ }_{K} V^{\prime}, v^{\prime}\right)$ are valued vector spaces such that $S\left({ }_{K} V, v\right) \equiv$ $S\left(K^{\prime} V^{\prime}, v^{\prime}\right)$. It follows that $K \equiv K^{\prime}$; consequently, $K$ and $K^{\prime}$ have a common prime field $F$, and $K \equiv K^{\prime}$ over $F$. Hence, $S\left({ }_{K} V, v\right) \equiv S\left(K_{K^{\prime}} V^{\prime}, v^{\prime}\right)$ over the skeleton $(F, \emptyset, \emptyset)$ of the trivial valued $F$-vector space $\left({ }_{F} 0, v\right)$. Since this space is trivially valuation disjoint from $K$ in $V$, we can apply Proposition 5.6 to obtain that $\left({ }_{K} V, v\right) \equiv\left({ }_{K}, V^{\prime}, v^{\prime}\right)$.

Since we have constructed the embeddings $f$ as liftings of the embeddings $f_{S}$, all results of this section remain valid if we formalise the valued vector spaces in a many-sorted structure so as to include their skeletons.

\subsection{Quantifier elimination}

As an immediate corollary to Proposition 5.6, we obtain:

Theorem 5.7 Let $(U, v) \subset(V, v)$ and $(U, v) \subset\left(V^{\prime}, v^{\prime}\right)$ be extensions of valued $K$ vector spaces. If $S(V, v)$ and $S\left(V^{\prime}, v^{\prime}\right)$ are elementarily equivalent over $S(U, v)$, then $(V, v)$ and $\left(V^{\prime}, v^{\prime}\right)$ are elementarily equivalent over $U$. In other words: valued $K$ vector spaces admit quantifier elimination relative to their skeletons in a language of valued vector spaces with fixed base field $K$.

For valued vector spaces with variable base field, it seems that there is no quantifier elimination in a natural "small" language. For instance, it does not suffice to adjoin predicates for $K$-valuation dependence:

$$
P\left(x_{1}, \ldots, x_{n}\right): \Leftrightarrow \exists k_{1}, \ldots, k_{n} \in K: v\left(k_{1} x_{1}+\ldots+k_{n} x_{n}\right)>\min _{\left\{i ; k_{i} \neq 0\right\}} v\left(x_{i}\right) .
$$


The reason is that if the latter holds, then we have no control on $v\left(k_{1} x_{1}+\ldots+k_{n} x_{n}\right)$. In particular, this value need not lie in $v(U)$ if $x_{1}, \ldots, x_{n}$ are taken from an $F$ subspace $U$ with $F \neq K$. So even if $\left({ }_{F} U, v\right)$ is a substructure of two valued vector spaces $\left({ }_{K} V, v\right)$ and $\left({ }_{K}{ }^{\prime} V^{\prime}, v^{\prime}\right)$ in the language of valued vector spaces enriched by the above predicates, we only know that $x_{1}, \ldots, x_{n}$ are $K$-valuation dependent in $V$ if and only if they are $K^{\prime}$-valuation dependent in $V^{\prime}$, but the values of their $K$-linear combinations in $V$ may differ significantly from those of their $K^{\prime}$-linear combinations in $V^{\prime}$.

\subsection{Some remarks on the model theory of skeletons}

Since Theorem 1.1 relates completeness and model completeness of elementary classes of valued vector spaces with completeness and model completeness of the elementary classes of their skeletons, we shall give some criteria for the latter. Two $K$-vector spaces of the same cardinality $>$ card $(K)$ are isomorphic over every common $K$-subspace of lower cardinality. One can use this fact to show the following results by an easy analogue of the back and forth construction in the proof of Proposition 5.6.

Lemma 5.8 An $\mathcal{L}$-elementary class $\mathcal{S}$ of infinite-dimensional vector spaces is complete if and only if the elementary class $\mathcal{K}=\left\{K ;{ }_{K} V \in \mathcal{S}\right\}$ of their base fields is complete. The $\mathcal{L}^{K}$-elementary class of all infinite $K$-vector spaces is complete and admits quantifier elimination.

Note that in the language $\mathcal{L}$ it can be expressed whether a vector space has infinite dimension or a fixed finite dimension. This is not possible in $\mathcal{L}^{K}$; there, it can only be expressed whether a space is infinite or (if the base field is finite) has a fixed finite cardinality. Again by a back and forth construction using the above stated properties of vector spaces, one shows:

Proposition 5.9 Let $\mathcal{K}$ be an elementary class of fields and $\mathcal{O}$ an elementary class of totally ordered sets.

1) The $\mathcal{L}_{\mathrm{S}}$-elementary class of skeletons $\left(K, \Gamma,\left\{B_{\gamma} ; \gamma \in \Gamma\right\}\right)$ with $K \in \mathcal{K}, \Gamma \in \mathcal{O}$ and infinite-dimensional $K$-vector spaces $B_{\gamma}$ is complete if and only if $\mathcal{K}$ and $\mathcal{O}$ are complete.

2) The $\mathcal{L}_{\mathrm{S}}^{K}$-elementary class of skeletons $\left(K, \Gamma,\left\{B_{\gamma} ; \gamma \in \Gamma\right\}\right)$ with $\Gamma \in \mathcal{O}$ and infinite $K$-vector spaces $B_{\gamma}$ is complete (or model complete, or admits quantifier elimination) if and only if $\mathcal{O}$ has the same property.

It is also possible to introduce predicates for $K$-dependence in vector spaces. For the correspondingly expanded theories, one obtains criteria for model completeness analogous to the first assertions in the above lemma and proposition. But this requires some formalism, and we leave the details to the reader.

As a consequence of Corollary 1.2 and the foregoing proposition, we obtain:

Theorem 5.10 1) Suppose that $\mathcal{V}$ is an $\mathcal{L}_{\mathrm{V}}$-elementary class of valued vector spaces whose components are all infinite-dimensional. Then $\mathcal{V}$ is complete if and only if the elementary class $\mathcal{K}=\left\{K ;\left({ }_{K} V, v\right) \in \mathcal{V}\right\}$ of their base fields and the elementary class $\left\{v(V) ;\left({ }_{K} V, v\right) \in \mathcal{V}\right\}$ of their value sets is complete. 
2) Suppose that $\mathcal{V}$ is an $\mathcal{L}_{\mathrm{V}}^{K}$-elementary class of valued $K$-vector spaces whose components are all infinite. Then $\mathcal{V}$ is complete (or model complete, or admits quantifier elimination) if and only if $\{v(V) ;(V, v) \in \mathcal{V}\}$ has the same property.

Since the class of dense linear orderings without endpoints is complete and admits quantifier elimination, Theorem 1.3 is an immediate consequence of this theorem.

\subsection{Ax-Kochen-Ershov Principles with additional predicates}

Suppose that we enrich the language of valued vector spaces by a set of predicates $P_{\mathrm{V}}:=\left\{P_{\mathrm{V}}^{i} ; i \in I\right\}$ and the language of skeletons by a set of predicates $P_{\mathrm{S}}:=\left\{P_{\mathrm{S}}^{j} ; j \in J\right\}$. Throughout this section, we let $\mathcal{T}$ be an elementary theory of valued vector spaces with skeletons and coefficient map in the enriched language $\mathcal{L}_{\mathrm{VS}} \cup P_{\mathrm{V}} \cup P_{\mathrm{S}}$, in which the $P_{\mathrm{V}}^{i}$ are interpreted as predicates on the valued vector spaces, and the $P_{\mathrm{S}}^{j}$ as predicates on the skeletons. We consider models $\left({ }_{K} V, v, P_{\mathrm{V}}, \pi, S\left({ }_{K} V, v\right), P_{\mathrm{S}}\right)$ of $\mathcal{T}$. If the predicates $P_{\mathrm{V}}^{i}$ are $\mathcal{T}$-definable from the $P_{\mathrm{S}}^{j}$, then it follows that an embedding $f$ of $\left({ }_{K} V, v\right)$ will respect the predicates $P_{\mathrm{V}}^{i}$ if the embedding $f_{S}$ of $S\left({ }_{K} V, v\right)$ respects the predicates $P_{S}^{j}$. Conversely, if the predicates $P_{\mathrm{S}}^{j}$ are $\mathcal{T}$-definable from the $P_{\mathrm{V}}^{i}$, then it follows that an embedding $f$ will respect the predicates $P_{\mathrm{V}}^{i}$ only if $f_{S}$ respects the predicates $P_{\mathrm{S}}^{j}$. In the above proofs of our model theoretic results, we can replace all embeddings by embeddings which preserve the respective predicates. In this way, we obtain the following versions for additional predicates:

Theorem 5.11 Suppose that $\mathcal{T}$ is as described above and that $\left({ }_{F} U, v, P_{\mathrm{V}}, \pi, \ldots\right) \subset$ $\left({ }_{K} V, v, P_{\mathrm{V}}, \pi, \ldots\right)$ is an extension of models of $\mathcal{T}$. If the predicates $P_{\mathrm{V}}^{i}$ are $\mathcal{T}$ definable from the predicates $P_{\mathrm{S}}^{j}$ and if $\left(S\left(_{F} U, v\right), P_{\mathrm{S}}\right) \prec_{\exists}\left(S\left({ }_{K} V, v\right), P_{\mathrm{S}}\right)$ in $\mathcal{L}_{\mathrm{S}} \cup P_{\mathrm{S}}$, then $\left({ }_{F} U, v, P_{\mathrm{V}}\right) \prec_{\exists}\left({ }_{K} V, v, P_{\mathrm{V}}\right)$ in $\mathcal{L}_{\mathrm{V}} \cup P_{\mathrm{V}}$. Conversely, if the predicates $P_{\mathrm{S}}^{j}$ are $\mathcal{T}$-definable from the predicates $P_{\mathrm{V}}^{i}$ and if $\left.{ }_{F} U, v, P_{\mathrm{V}}\right) \prec_{\exists}\left({ }_{K} V, v, P_{\mathrm{V}}\right)$ in $\mathcal{L}_{\mathrm{V}} \cup P_{\mathrm{V}}$, then $\left(S\left(_{F} U, v\right), P_{\mathrm{S}}\right) \prec_{\exists}\left(S\left({ }_{K} V, v\right), P_{\mathrm{S}}\right)$ in $\mathcal{L}_{\mathrm{S}} \cup P_{\mathrm{S}}$.

Analogous assertions hold for " $\prec$ " and "三” in the place of " $\prec_{\exists}$ ". For valued $K$-vector spaces (with the languages $\mathcal{L}_{\mathrm{V}}^{K} \cup P_{\mathrm{V}}$ and $\mathcal{L}_{\mathrm{S}}^{K} \cup P_{\mathrm{S}}$ in the place of $\mathcal{L}_{\mathrm{V}} \cup P_{\mathrm{V}}$ and $\mathcal{L}_{\mathrm{S}} \cup P_{\mathrm{S}}$ ), we also have the analogue to assertion 4) of Theorem 1.1.

We leave it to the reader to write down the corresponding versions of Corollary 1.2 and Theorem 1.3.

\subsection{Ordered $(K,<)$-vector spaces}

In the following, let $(K,<)$ be any ordered field.

Proposition 5.12 Let $(U,<) \subset(V,<)$ and $(U,<) \subset\left(V^{*},<^{*}\right)$ be extensions of ordered $(K,<)$-vector spaces. Assume that $\left(V^{*},<^{*}\right)$ is $\kappa$-saturated, with $\kappa>$ card $(V)$. Then there is an embedding of $(V,<)$ in $\left(V^{*},<^{*}\right)$ over $U$.

Proof: As in the proof of Proposition 5.2, we can assume that $V=U+K y$. Consider the following set of $\mathcal{L}_{\mathrm{OV}}^{K}(U \cup\{X\})$-sentences, where $X$ is a new constant symbol:

$$
p=\left\{" X<_{V} b " ; b \in U \text { and } y<b\right\} \cup\left\{\text { "c }<_{V} X " ; c \in U \text { and } c<y\right\}
$$


We show that $p \cup \operatorname{diagram}(U,<)$ is consistent. If $p=\emptyset$, then there is nothing to show. Assume that $p \neq \emptyset$, which yields that $U \neq 0$. We let $p_{0} \subset p$ be nonempty and finite and show that $p_{0}$ is realisable in $(U,<)$. The sets $p_{0}^{<}:=\left\{b \in U ;{ }^{\prime} X<_{V}\right.$ $\left.b " \in p_{0}\right\}$ and $p_{0}^{>}:=\left\{c \in U ; " c<_{V} X " \in p_{0}\right\}$ are finite; so let $b_{0}$ the minimum of the first set and $c_{0}$ the maximum of the second set. Then $c_{0}<b_{0}$ because $c_{0}<y<b_{0}$ in $V$. Since every nontrivial ordered $(K,<)$-vector space is densely ordered, there is an element $a \in U$ such that $a_{0}<a<b_{0}$. Now $a$ realises $p_{0}$ because $a<b_{0} \leq b$ for every $b \in p_{0}^{<}$and $c \leq c_{0}<a$ for every $c \in p_{0}^{>}$.

By the saturation property of $\left(V^{*},<^{*}\right), p$ is realised by some $y^{*} \in V^{*}$. We define:

$$
f(k y+b)=k y^{*}+b \text { for every } k \in K, b \in U .
$$

It is clear that $\left.f\right|_{U}=\mathrm{id}$ and that $f$ is additive and preserves scalar multiplication. Let us show that $f$ preserves the order (and hence is injective), that is,

$$
0<k y+b \Leftrightarrow 0<k y^{*}+b .
$$

This is clear if $k=0$; so take $k \neq 0$. If $0<k$, then $0<k y+b \Leftrightarrow 0<y+k^{-1} b \Leftrightarrow$ " $-k^{-1} b<_{V} X$ " $\in p \Leftrightarrow 0<y^{*}+k^{-1} b \Leftrightarrow 0<k y^{*}+b$. If $k<0$, then $0<$ $k y+b \Leftrightarrow y+k^{-1} b<0 \Leftrightarrow$ "X $X<_{V}-k^{-1} b$ " $\in p \Leftrightarrow y^{*}+k^{-1} b<0 \Leftrightarrow 0<k y^{*}+b$. This completes our proof.

Again by model theoretic back and forth constructions similar to the one used in the proof of Proposition 5.6, we obtain:

Corollary 5.13 If $(U,<) \subset(V,<)$ and $(U,<) \subset\left(V^{\prime},<^{\prime}\right)$ are extensions of ordered $(K,<)$-vector spaces, then $(V,<)$ and $\left(V^{\prime},<^{\prime}\right)$ are elementarily equivalent over $U$.

Proposition 5.14 Let $\mathcal{O}$ be an elementary class of totally ordered sets and $(K,<)$ an ordered field. Then the elementary class of ordered skeletons $\left(K, \Gamma,\left\{B_{\gamma} ; \gamma \in \Gamma\right\}\right)$ with $\Gamma \in \mathcal{O}$ and ordered $(K,<)$-vector spaces $B_{\gamma}$ is complete (or model complete, or admits quantifier elimination) if and only if $\mathcal{O}$ has the same property.

Theorem 1.6 is a direct consequence of Corollary 5.13. Since the components of ordered $(K,<)$-vector spaces with compatible valuation are again ordered $(K,<)$ vector spaces, Proposition 5.14 together with Corollary 1.5 proves Theorem 1.7.

\section{References}

[C-K] Chang, C. C. - Keisler, H. J.: Model Theory, Amsterdam (1973)

[D] van den Dries, L.: Quantifier elimination for linear formulas over ordered and valued fields, Bull. Soc. Math. Belg. 23 (1981), 19-32

[F] Fuchs, L.: Abelian p-groups and mixed groups, Montréal (1980)

[K1] Kuhlmann, S.: On the structure of nonarchimedean exponential fields I, Arch. Math. Logic 34 (1995), 145-182

[K2] Kuhlmann, S.: Valuation Bases for Extensions of Valued Vector Spaces, to appear in Forum Math. (1995)

[K3] Kuhlmann, S.: Infinitary Properties of Valued and Ordered Vector Spaces, submitted

[KF] Kuhlmann, F.-V.: Valuation Theory of Fields, Abelian Groups and Modules, to appear in the "Algebra, Logic and Applications" series, eds. Göbel and Macintyre, Gordon \& Breach, New York. 